MACROMOLECULAR ORGANIZATION OF HEMOCYANINS AND APOHEMOCYANINS

AS REVEALED BY ELECTRON MICROSCOPY

H. Fernández-Morán, E. F. J. van Bruggen, and M. Ontsuki

Department of Biophysics

The University of Chicago

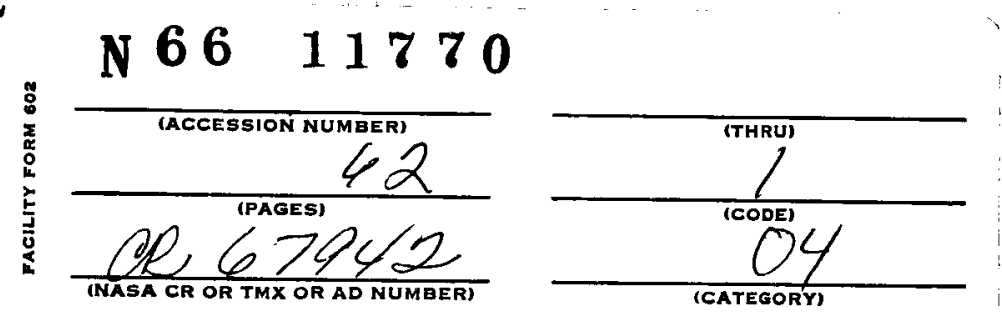

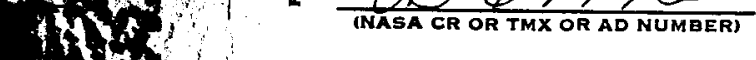

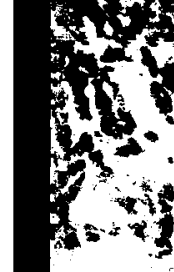

$18:$ :

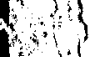


MACROMOLECULAR ORGANIZATION OF HEMOCYANINS

AND APOHEMOCYANINS AS

REVEALED BY ELECTRON MICROSCOPY

H. Fernández-Morán, E. F. J. van Bruggen*, and M. Ohtsuki

Department of Biophysics

The University of Chicago

This work was supported by Grants: NB-04267 and GM-13243 from The National Institutes of Health, by Grant NSG 44I-63 of The National Aeronautics and Space Administration, by United States Atomic Energy Commission Contract AT (11-1)-1344, by the L. Block Fund, and by the otho Sprague Memorial Fund of The University of Chicago.

* International Postdoctoral Research Fellow of The United States Public Health Service Grant: 1 FO5-TW824-01.

Permanent address: Laboratorium voor Structuurchemie, Bloemsingel 10, Groningen, The Netherlands. 


\section{Macromolecular Organization of Hemocyanins and Apohemocyanins as \\ Revealed by Electron Microscopy}

\section{SUMMARY}

Comparative electron microscopic studies of the structural organization of representative hemocyanins and apohemocyanins from Mollusca and Arthropoda are described. Helix pomatia, Busycon canaliculatum, and Loligo pealei were chosen as examples of the Mollusca; Homarus americanus and Limulus polyphemus represented the Arthropoda. Mollusca hemocyanins are cylindrical molecules (diameter about $340 \AA$, height ranging from $140 \AA$ to $680 \AA$ ) built up from 3 to 12 rows of subunits. Arthropoda hemocyanins are built from a cubic monomer (105 $\AA$ ) in various stages of organization which is species dependent. Mollusca hemocyanins are distinctly different from Arthropoda hemocyanins, although they seem to be built from analogous subunits. Observations indicating the possible presence of certain constituents specifically localized in the core of the Mollusca hemocyanins are discussed. A differentiated outer layer is regularly found around the hemocyanin and apohemocyanin molecules of Mollusca. It is not known whether these structures are actual components of the native molecules or are determined by the preparation techniques. Their possible presence can have significant biological implications. Reproducible differences between hemocyanins and apohemocyanins were observed only in the Mollusca and under certain conditions. High resolution electron microscopy using improved preparation techniques and instrumentation (coherent microbeam illumination) revealed new structural details in the molecules close to quaternary levels. The results are discussed in relation to the avilable biochemical and biophysical data on these highly organized macromolecular complexes. 


\begin{abstract}
Macromolecular organization of hemocyanins and apohemocyanins as revealed by electron microscopy. H. Fernández-Morán, E. F. J. van Bruggen \& M. Ohtsuki.

J. Mol. Biol.

(Dept. of Biophys., University of Chicago, Chicago, III., U. S. A. ).

Comparative high resolution electron microscopic studies of the structural organization of representative hemocyanins and apohemocyanins from Mollusca and Arthropoda are described. Mollusca hemocyanins are cylindrical molecules (diameter about $340 \AA$, height ranging from $140 \AA$ to $680 \AA$ ) built up from 3 to 12 rows of subunits. Arthropoda hemocyanins are built from a cubic monomer (105 $\AA$ ) in various stages of organization which is species dependent. Mollusca hemocyanins are distinctly different from Arthropoda hemocyanins, although they seem to be built from analogous subunits. New structural details observed close to the quaternary levels are discussed in relation to available biochemical and biophysical data on these highly organiz ed macromolecular complexes.
\end{abstract}




\section{Macromolecular Organization of Hemocyanins \\ and Apohemocyanins as \\ Revealed by Electron Microscopy}

\section{Introduction}

Hemocyanins are important oxygen-transporting blood proteins which occur freely dissolved in the hemolymph of certain invertebrates of phylums Mollusca and Arthropoda (Redfield, 1934; Dawson \& Mallette, 1945). With regard to their physiological function, hemocyanins are similar to hemoglobin, the respiratory pigment in the blood of higher animals. There is also a certain analogy with the erythrocmorins (Roche, Bessis \& Thiery, 1960a, 1960b; Roche, Bessis, Breton-Gorius \& Stralin, 1961; Levin, 1963a), the chlorocruorins, and haemerythrin (Redfield, 1934; Klotz \& Klotz, 1955) which are oxygen-transporting proteins of some invertebrate species. However, only hemocyanins contain copper (0.18\% to $0.25 \%)$, which is probably univalent. One molecule of oxygen is bound for every two atoms of copper (Redfield, 1934).

Eriksson-Quensel \& Svedberg (1936) have studied hemocyanins extensively in the ultracentrifuge under different conditions. The molecules were shown to dissociate reversibly into smaller units depending upon the $\mathrm{pH}$, ionic strength, etc. Undissociated molecules of different biological origin vary widely in size. Their molecular weights range from several hundred thousands in the case of certain Arthropoda to several millions for certain Mollusca.

Earlier studies (Stanley \& Anderson, 1942; Quaife \& Baylor, 1943; Polson \& Wyckoff, 1947; Schramm \& Berger, 1952; and Lauffer \& Swaby, 1955) were confirmed and extended by systematic correlated electron microscope studies by van Bruggen (1962; 1964 ) and van Bruggen, Wiebenga \& Gruber (1962a; 1962b; 1963). 
These recent studies, mainly using negative staining techniques, showed that Mollusca hemocyanin molecules are roughly cylindrical in shape. The diameter of the cylinders is about $300 \AA$; the length varies between $175 \AA$ and $2,000 \AA$, depending upon the species. A tentative model was proposed for Helix pomatia (Roman snail) hemocyanin. Arthropod hemocyanin exhibits a different structure; its basic unit seems to be a cube with an edge of about $80 \AA$. They are observed as monomers, dimers, tetramers, etc., of this basic unit (van Bruggen et al. 1963; van Bruggen 1964).

The size of hemocyanin molecules and the ease of preparation from fresh blood without extensive manipulations makes these organized respiratory pigments ideal subjects for electron microscopy. Moreover, they can be subjected to controlled experimental biophysical, biochemical and physical chemical modifications which can be correlated with the results of electron microscopy.

It is possible to remove the copper from a hemocyanin molecule (Kubowitz, 1938) to obtain apohemocyanin. Irvestigations of any structural changes thay may occur, using high resolution electron microscopy and possibly electron diffraction, may yield information relevant to the localization of the copper that plays a key role in this molecules.

Improved preparation techniques (Hall, 1955; Huxley, 1956; Brenner \& Horne, 1959; Fernández-Morán, 1960a) and instrumentation (Fernández-Morán, 1960b;, 1964) for high resolution electron microscopy have permitted direct visualization of the substructure of viruses (Brenner \& Horne, 1959; Caspar \& Klug, 1962) and of certain multienzyme complexes (Fernández-Morán, Reed, Koike \& Willms, 1964). These results can be correlated with the available X-ray data and biochemical information to deduce the actual arrangement of the protein subunits and molecular organization of these systems. It 
therefore appeared worthwhile to attempt a similar correlated approach, in the study of hemocyanins and apohemocyanins, which would lead to a more detailed model of their organization at a level of resolution close to the quaternary structure of the constituent proteins.

This paper reports on comparative studies of the structural organization of representative hemocyanins and apohemocyanins from Mollusca and Arthropoda as revealed by high resolution electron microscopy using a variety of supplementary techniques. Helix pomatia, Busycon canaliculatum, and Loligo pealei were selected as examples of the Mollusca; Homarus americanus and Limulus polyphemus as examples of the Arthropoda. The results will be discussed in relation to the available biochemical and biophysical data.

Additional investigation on the reassembling of mixtures of the subunits of two different hemocyanins after dissociation of the molecules at high $\mathrm{pH}$ was done with a mixture of Helix and Loligo hemocyanins, and with a mixture of Helix and Limulus hemocyanins. The results of these investigations are reported in a companion paper.

2. Materials

(a) Hemocyanin

The hemolymphs of Busycon canaliculatum, Loligo pealei, and Iimulus polyphemus were collected fresh and sent refrigerated from the Marine Biological Laboratory at Woods Hole, U.S.A. Helix pomatia hemolymph was collected in the usual way by heart puncture, kept under toluene at $+4^{\circ} \mathrm{C}$ and sent from the Laboratory of Structural Chemistry at Groningen, The Netherlands, on ice in a dewar flask. Homarus americanus hemolymph was collected locally by bleeding a living animal after extraction of a leg. In this case a fibrin-like component formed by shaking was removed by filtration.

$$
-4-
$$


In most cases the hemolymph was studied without further purification, since the blood does not contain appreciable amounts of other proteins besides hemocyanin. In a few cases the hemolymphs were dialysed first against a solution containing $0.01 \mathrm{M}$-ammonium acetate and $0.0005-0.01 \mathrm{M}$ calcium acetate ( $\mathrm{pH}$ between 6 and 7 ).

\section{(b) Apohemocyanin}

Apohemocyanin (copper-free hemocyanin) was prepared by the method of Ghiretti (1956) by dialysis of the hemolymph against several changes of a solution containing $0.01 \mathrm{M}-$ $\mathrm{KCN}, 0.01 \mathrm{M}$-borax, and $0.01 \mathrm{M}-\mathrm{CaCl}_{2}$ at $\mathrm{pH}$ 8.2. The manipulations were carried out in a fume hood at $21^{\circ} \mathrm{C}$. The $\mathrm{KCN}$ was removed by dialysis against several changes of a solution containing $0.01 \mathrm{M}$-ammonium acetate and $0.01 \mathrm{M}$-calcium acetate brought to $\mathrm{pH} 6$ to 7 with dilute acetic acid. The entire preparation procedure for apohemocyanin was carried out under the same conditions as those reported earlier (van Bruggen et al., 1962). At that time the copper content was determined spectrophotometrically as a carbamate complex by combining the procedures of Peterson and Bollier (1955) and Sandell (1944). Helix pomatia hemocyanin contained $0.21 \% \mathrm{Cu}$, Helix pomatia apohemocyanin contained $0.05 \% \mathrm{Cu}$. The same procedure was followed throughout for the preparations of solutions of apohemocyanin from other types of hemocyanin (Busycon, Loligo, Limulus, Homarus).

(c) Buffers

The hemocyanin and apohemocyanin solutions were diluted with buffer for the electron microscopy. The following buffers were the main ones used:

(i) 0.01 M-ammonium acetate ( $\mathrm{pH} 6.8$ )

(ii) $0.01 \mathrm{M}$-acetic acid/ammonium acetate $+0.0005 \mathrm{M}$ to $0.01 \mathrm{M}-\mathrm{Ca}-a c e t a t e$ and/or $\mathrm{Mg}$-acetate ( $\mathrm{pH}$ between 6 and 7). (iii) $0.01 \mathrm{M}-\left(\mathrm{NH}_{4}\right)_{2} \mathrm{CO}_{3} / \mathrm{NH}_{4} \mathrm{OH}$ (pH between 10 and $1 \mathrm{l}$ ). 
The final protein concentration was very much dependent upon the specimen preparation method chosen, and varied from $50 \mathrm{\mu g}$ to $800 \mu \mathrm{g} / \mathrm{ml}$ buffer.

The water used for all the preparation was de-ionized, led over charcoal, double-distilled in a quartz distillation unit and ultra-filtered through a $100 \AA$ millipore filter. The solutions were filtered through bacterial filters.

\section{Methods}

(a) Specimen Preparation

Several variations of the negative staining technique (Hall, 1955; Huxley, 1956; Brenner \& Horne, 1959) and of positive staining were used.

(i) Preparation of a fenestrated formvar film covered with an ultrathin carbon film.

Specimen grids of copper, titanium and platinum were usually coated with fenestrated formvar films (Fernández-Morán \& Finean, 1957) with uniformly distributed holes of $100 \AA$ to 5,000 $\AA$ diameter. These fenestrated films were prepared by coating clean glass slides or freshly cleaved mica with a $0.1 \%$ or $0,2 \%$ solution of formvar in 1 , 2-dichloroethane in an atmosphere of precisely controlled relative humidity ( $80 \%$ to 90\%). These films were stabilized by vacuum evaporation of a carbon coat. Best results were obtained by covering these fenestrated films with extremely thin carbon films (thickness of $10 \AA$ to $50 \AA$ approximately) which are very resistant, practically structureless and superior to the standard ultrathin formvar films. These ultrathin carbon films were prepared by evaporation on freshly cleaved mica from spectroscopically pure carbon pointed rods from a distance of about $12 \mathrm{~cm}$. A Varian high-vacuum evaporation unit was used (Varian ultra-high vacuum unit Model 922 with vacion pump 921) with specially adapted liquid nitrogen and liquid helium attachments. This unit has vacsorption fore -6- 
pumps, and therefore is devoid of all oil vapors and attains a vacuum of $10^{-8}$ to $10^{-9} \mathrm{~mm} \mathrm{Hg}$. which can be monitored continuously with a recording Alpert gauge. The carbon films that are evaporated in this atmosphere free of residual gases are distinctly superior to the standard carbon films prepared in pumping units with diffusion pumps. After evaporation the films can be floated off the mica in ultra-filtered, double quartz-distilled water. The carbon films resemble monomolecular films, and require special oblique illumination because they are barely visible. They can be picked up on the carboncoated fenestrated formvar films and afford, after drying, ideal substrates for high resolution electron microscopy.

(ii) Preparation of fenestrated formvar films with asbestos. In some cases, fenestrated films were used without any supporting film (Huxley \& Zubay, 1960; Fernández-Morán, 1962a). A useful variant of this procedure consists of bridging the holes of the fenestrated film with suitably dispersed ultra-thin filaments of asbestos (Chrysotile) prior to deposition of the negative staining film. The asbestos fibers act as reinforcing structures to bridge the larger holes, and an extremely thin film of the negative stain is frequently found wedged between the hole contours and the asbestos filament. This technique, which is described separately (Fernández-Morán, in preparation) has the additional advantage that the asbestos fibers exhibit a characteristic periodic lattice, parallel to the long axis, with a period of $7.3 \AA$ which can serve as an internal calibration standard.

\section{(iii) Preparation of fenestrated film with bentonite.}

An additional variation for mounting negatively and positively stained specimens utilizes extremely thin ( $10 \AA$ to $20 \AA$ ) bentonite and montmorillonite particles prepared by the method of McLaren and Peterson (1961). Such particles adsorb the hemocyanin molecules and can then be used to bridge the gaps in fenestrated films. 
(iv) Preparation of the protein.

The following preparation techniques were used:

(a) A droplet of the hemocyanin or apohemocyanin solution diluted with one of the buffers described above to a protein concentration of $25 \mu \mathrm{g} / \mathrm{ml}$, was placed on the specimen grid with a fine, freshly drawn glass pipette. After one to two minutes the excess portion of the solution was removed by floating the grid upside down on a solution of the corresponding buffer alone, for about fifteen seconds. In some cases the specimen was then fixed by transporting the grid to a fixing solution in a freshly flamed platinum loop. For fixation we used $1 \%$ or $3 \%$ formaldehyde neutralized with $\mathrm{KOH}$ or $1.5 \%$ glutaraldehyde in $0.01 \mathrm{M}$-phosphate buffer ( $\mathrm{pH} 7.0$ ). The fixation was done on ice $\left(0^{\circ} \mathrm{C}\right)$ for thirty minutes, after which the specimens were rinsed by floating them on buffer alone for about fifteen seconds.

Both fixed and unfixed specimens were stained by floating on the staining solution for one, two or five minutes. Finally each specimen was air dried by blotting the edge of the grid to a piece of filter paper until nearly dry.

This method is very similar to the one first described by Huxley (1963). It was developed in this form by van Bruggen et al. for the examination of sucrose and glycerol containing gradient material.

The following solutions were used for negative staining: 1) $0.5 \%$ and $2 \%$ solutions of phosphotungstic acid, silicotungstic acid or boro tungstic acid neutralized with $\mathrm{KOH}$ or $\mathrm{NaOH}$ to $\mathrm{pH} 7.2$.

2) $1 \%$ and $2 \%$ solutions of ammonium metatungstate. 3) $0.5 \%, 1 \%$, and $2 \%$ solutions of "uranium-EDTA" (van Bruggen et a1., 1962a) or uranium citrate in $0.01 \mathrm{M}$-ammonium acetate brought to $\mathrm{pH} 7$ to $10 \mathrm{w}$ 1th ammonia. For positive staining $1 \%$ and $2 \%$ solutions of unbuffered uranyl acetate were used. 
In order to prevent the formation of particles, all the staining solutions were freshly prepared and ultrafiltered in a manner described previously (Fernández-Morán, 1964).

(b) Microdroplet cross-spraying techniques were used (Fernández-Morán, 1962a, 1962b) by means of a multiple-spraying device provided with suitably arranged separate quartz capillaries (internal opening 0.2 to 0.1 millimeters) for specimens and for reagents. It is possible to obtain controlled, brief interaction of specimen microdroplets with microdroplets of $1 \%$ to $2 \%$ potassium phosphotungstate ( $\mathrm{pH} 7.2$ ), uranyl acetate, or other heavy metal solutions. The crosssprayed microdroplets (about 0.1 to $10 \mu$ in diameter) collide in mid-air and rapidly interact shortly before impinging on the specimen grid. As a result of the small size and relatively high speed of the cross-spraying microdroplets there is a favorable combination of rapid specimen cooling and limited drying to attain a high degree of preservation of labile structures (Fernández-Morán, 1962; 1964). This technique can also be modified to obtain cross-spraying of the unfixed specimens with $1 \%$ to $3 \%$ buffered glutaraldehyde solutions for fixation, thus preventing the aggregation which often occurs during fixation.

(c) A mixture of the protein with a concentration of about $400 \mathrm{\mu g} / \mathrm{ml}$ and of the stain was sprayed directly onto the carbon film, using the standard negative staining technique described by Brenner \& Horne (1959).

(d) A combination of negative staining and embedding with ultrathin sectioning was used (Fernández-Morán, 1952; 1962; 1964). Dried or frozen droplets of the hemocyanin solutions or pellets of the hemocyanin prepared by ultra-centrifugation were mounted on a Moran-Leitz ultramicrotome and sectioned with a diamond knife (Fernández-Morán, 1953). 
The pellets or suspensions were also sucked into thin plastic capillaries ( 0.1 to 0.2 millimeters inner diameter) using the technique described earlier for examination of oriented tobacco mosaic virus gels (Fernández-Morán \& Schramm, 1958). The specimen capillaries were cut into short segments and mounted on the microtome where they could either be sectioned directly or after freezing. These ultrathin sections are collected directly on a $1 \%$ to $2 \%$ buffered phosphotungstate solution or on unbuffered $1 \%$ to $2 \%$ urany 1 acetate and picked up on filmed specimen grids. Although this technique has inherent limitations, mainly caused by drying artifacts, it provides interesting information on the aggregates of hemocyanin molecules in the ultra-centrifugated pellets. The results will be reported in a separate publication.

(e) In a number of cases, it was found useful to combine positive and negative staining with uranyl acetate with shadow casting with carbon in an ultra-high vacuum of $10^{-8}$ to $10^{-9} \mathrm{~mm} \mathrm{Hg}$. This technique was particularly useful also when dealing with unstained fresh and fixed hemocyanin molecules, since it permits a determination of the geometry and contours of the molecule without the introduction of staining artifacts. Thanks to the high contrast obtained with microbeam illumination using pointed filaments, the carbon shadows could be made visible depicting extremely fine detail which would normally be obscured by the standard platinum or tungsten shadowing.

\section{Electron Microscopy}

The electron microscope studies were carried out in a facility which was designed to provide optimum conditions for consistent attainment of high resolution. The microscopes were provided with a highly regulated power supply (50 kilowatt motor generator set, specially designed and manufactured by Westinghouse Company, which is equipped with a new solid state regulator giving better than $0.1 \%$ $-10-$ 
voltage stability and very low harmonic distortion). The microscopes were mounted on vibration-free foundations in clean room environments.

A Siemens Elmiskop I and Elmiskop IA, and a Hitachi IlB microscope were used, operating mainly at $80 \mathrm{kV}$ (also at $100,75,60,50$, and $40 \mathrm{kV}$ for selected specimens).

All microscopes were provided with improved pointed filaments (Fernández-Morán, 1960b) of single-crystal tungsten with a tip radius of $1 \mu$ to $10 \mu$. These pointed filaments were used routinely with the double condenser system to provide intense microbeam illumination with high coherence and low angular divergence. Recent improvements in the gun design make it possible to attain image brightness(with a $1 \mu$ to $10 \mu$ spot size, beam current 1 to 8 micro-amperes at 75 to $80 \mathrm{kV}$ ) which is adequate for direct observation of image detail at the highest electron optical magnification. In most of the critical work, condenser apertures of $20 \mu, 50 \mu$ and $100 \mu$ were used in condenser II to obtain a greater transverse coherence length and enhance contrast in the images. Using multiple objective apertures of copper or platinum $(30 \mu$ and $50 \mu$ diameter) as well as very thin ( $5 \mu$ to $10 \mu$ ) molybdenum apertures of $30 \mu$ and $50 \mu$ the astigmatism of the objective lens could readily be corrected under direct observation and reduced to less than $0.1 \mu$. For high resolution studies the measured astigmatism of the objective lens was $0.05 \mu$ or less. One of the electron microscopes was provided with a separate, specially regulated power supply with reduced ripple for the objective lens, with improved controls permitting recording of through-focal series in steps of $80 \AA$ to $200 \AA$. All instruments were provided with special Viton gaskets to improve the high vacuum and reduce the residual gases. Most of the high resolution micrographs were recorded us ing liquid nitrogen cold stages and anti-contamination devices. A combination of low intensity microbeam illumination and controlled 
specimen cooling $\left(-80^{\circ}\right.$ to $\left.-180^{\circ} \mathrm{C}\right)$ was found to be very useful for the study of delicate structures of the hemocyanin molecules, as well as for controlled etching experiments.

The higher efficiency of the pointed filament sources enabled us to use Ilford high resolution plates routinely ( 4 to 20 seconds exposure at 40,000 to 160,000 X electron optical magnification). In addition, Kodak High Definition $70 \mathrm{~mm}$ films on a thin polyester base were found to be very useful for recording up to 90 exposures without breaking the vacuum. This was particularly valuable in recording through-focal series using the cold stages. Ilford $\mathrm{N}-40$ and Gavaert Scientia 23D50 plates were also routinely used.

For all critical measurements, calibration of the microscope was carried out at the time of recording the electron micrographs, using a diffraction grating replica $(54,864$ lines per inch and 28,800 lines per inch, supplied by Ladd Research Industries) in the same position as the grid. An additional check on the calibration was carried out by Ronald Luftig and Dr. Keiji Yada using combined electron microscopy and electron diffraction of selected planes from copper pthalocyanin and $\mathrm{K}_{2} \mathrm{PTCl}_{4}$ crystals.

Measurements were carried out directly on the plates and films using an optical comparator (Nikon Shadowgraph Model 6C).

The plates were developed with Agfa Rodinal, Kodak D72, or D76 under controlled temperature conditions $\left(20^{\circ} \mathrm{C}\right)$. The prints were made from the original plates or from contact duplicates taking special precautions to eliminate aberrations using the Durst S-45 enlarger with point source or with a mercury vapor lamp.

The observations described here are based on the evaluation of more than 2,000 plates and films in which an average resolution of $8 \AA$ to $15 \AA$ was consistently achieved. 
About 2,000 additional pictures, taken at the Laboratory of Structural Chemistry, University of Groningen, The Netherlands, with a Philips EM-200 electron microscope, were also evaluated.

4. Results

(a) Moliusca

(i) Helix pomatia hemocyanin

Negative staining. As shown in Plates I and III the hemocyanin molecules appear as circles of about $380 \AA$ (Histogram Fig. 1) and as rectangles in specimens negatively stained with "uranium-EDTA" as described by van Bruggen et al.(1962a). The circles contain subunits in characteristic five-fold or ten-fold symmetrical arrangement. In the rectangles the subunits are arranged in six parallel rows.

In good agreement with van Bruggen et al. (1962a; 1963) the size of the rectangles perpendicular to the rows is found to be about $400 \AA$ (Histogram Fig. 1) and the other sides show a variation from $350 \AA$ to $400 \AA$. There is a slight difference of about $10 \%$ to $15 \%$ between the dimensions of the diameter of the circles and the width of the rectangles. This is compatible with the assumption that the circles and rectangles are projections of the same cylindrical molecule, taking into consideration the alterations introduced by the preparation techniques.

Certain structural features which had not been clearly seen before are now discernible in greater detail, both in the fresh and in the fixed molecules using different preparation techniques. The new details relate mainly to the subunit structure, the internal configuration of the molecules, and to their outer coats.

In the center of the circles, in specimens negatively stained with phosphotungstate by the double-spray method, (Plate II) 

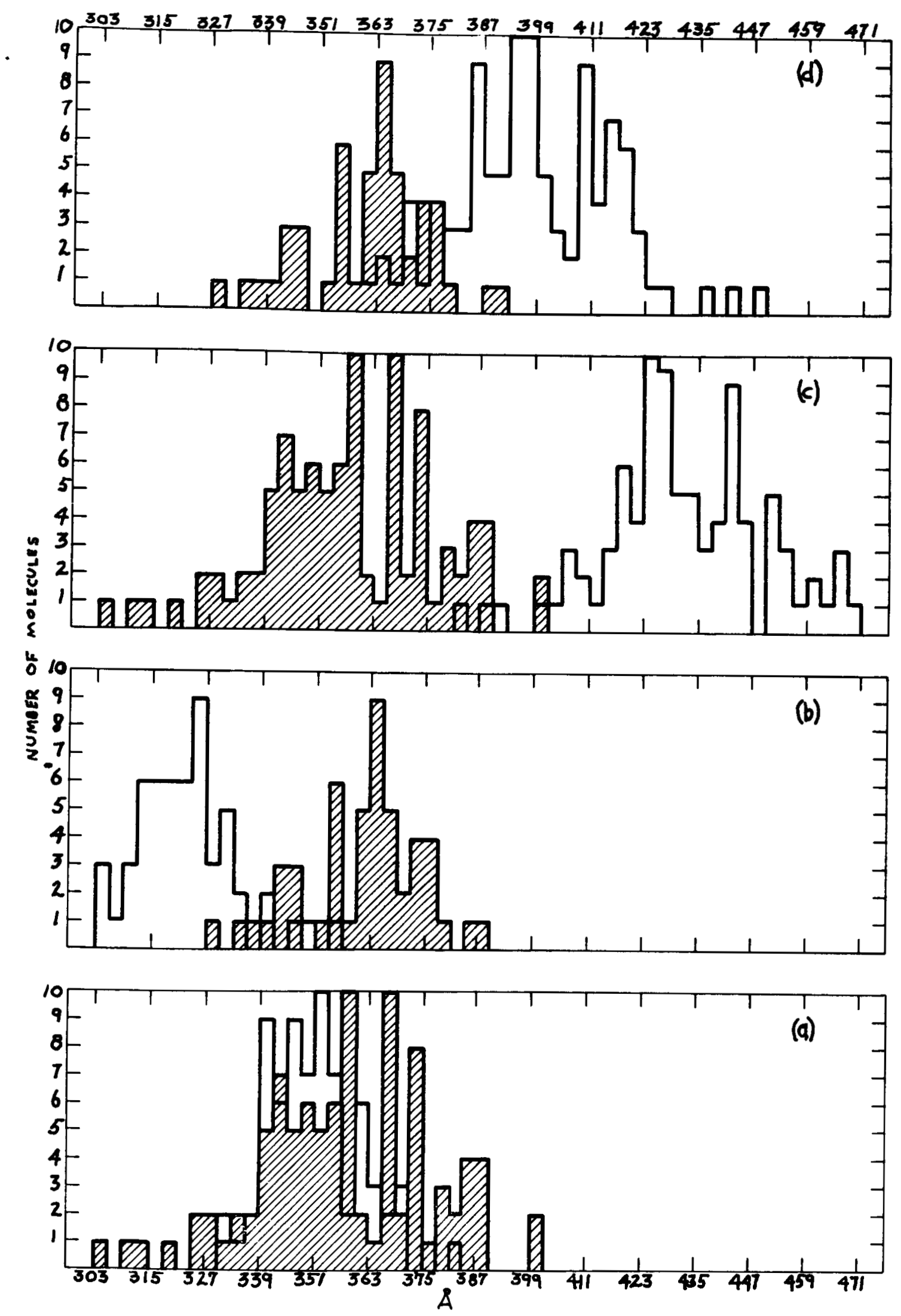

Size distribution of circular and rectangular forms of Helix pomatia hemocyanin molecules negatively stained with phosphotungstate (PTA), uranyl acetate (UA) and uranium EDTA (UE). (a) and (c) length of the rectangular forms measured perpendicular to rows: PTA - clear areas in (a), UA - clear areas in (b), UA - hatched areas, UE - clear areas in (d). 


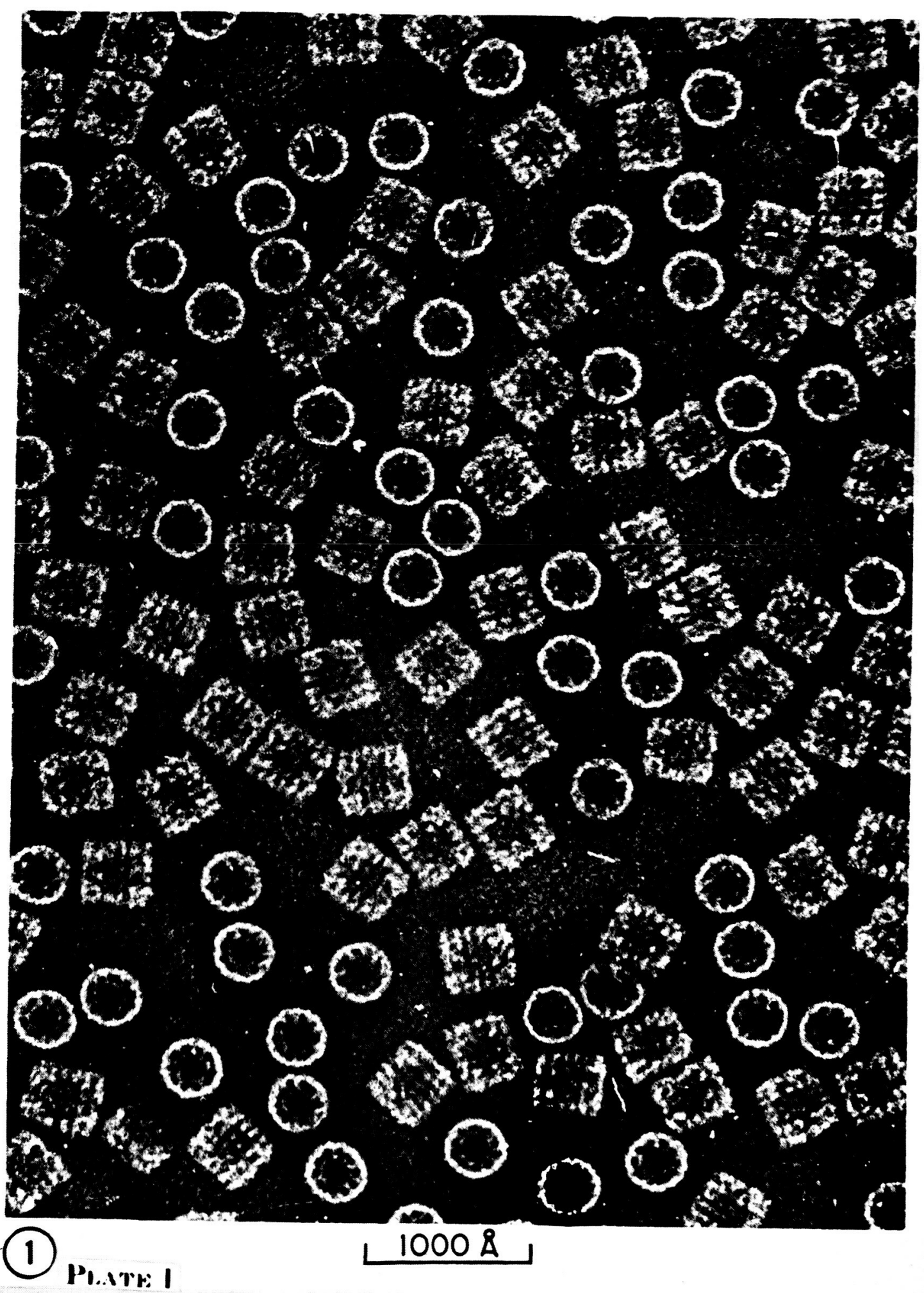

Electron micrograph of typical preparation of Hellx pomatia hemocyanin molecules negatively stained with a "uranium-EDTA" complex without prevlous fixation. The circular forms oontain subunits in ten-fold symmetrical arrangement; the subunits in the rectangular forms are disposed in six parallel rows. X 264,000. 


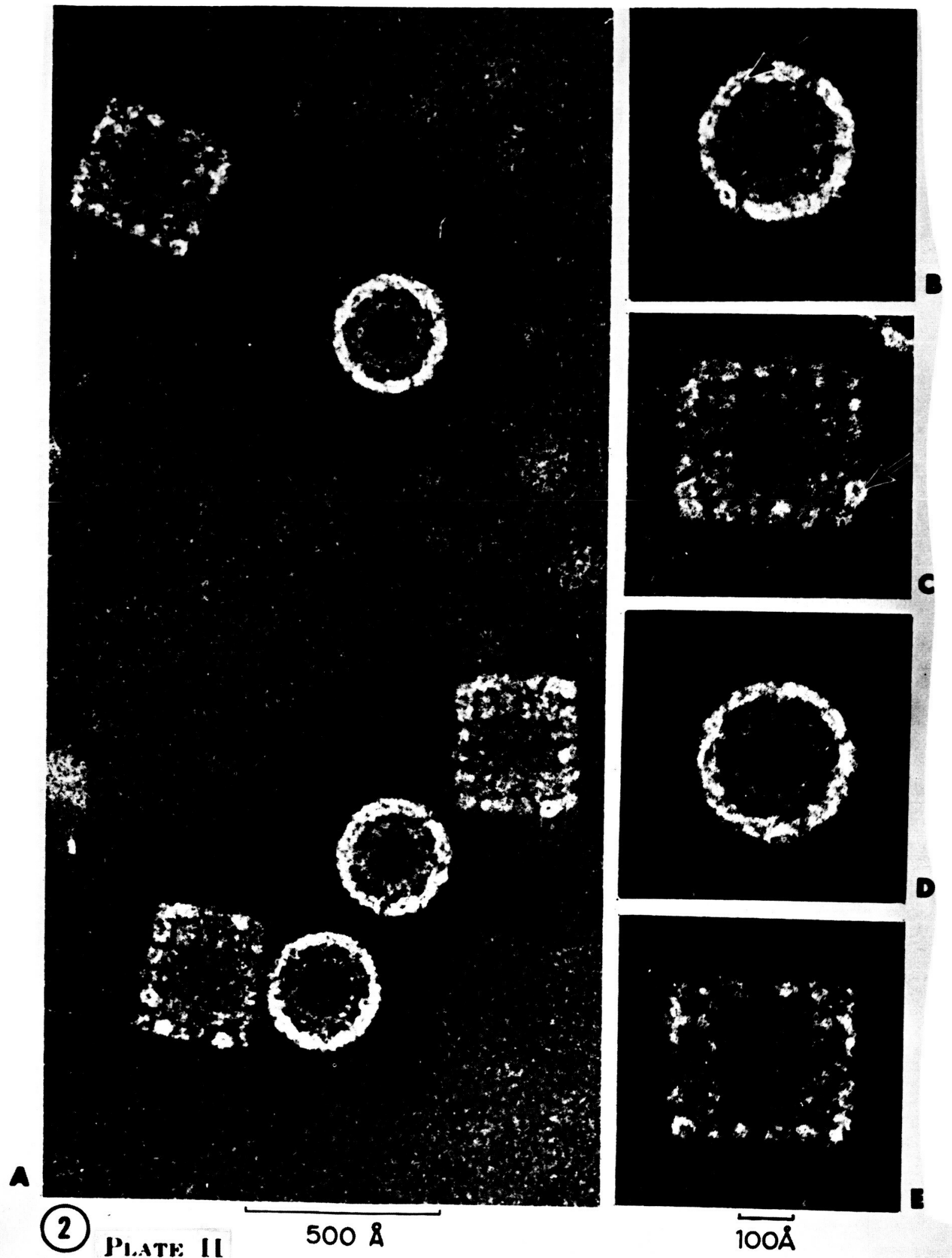

Helix pomatia hemocyanin molecules negatively stained with potassium phosphotungstate by the double-spray method without previous fixation. In the circular forms a semi-opaque region surrounds the dense core, followed by a concentric dense middle ring, a clear annular zone with a fine central layer $(a r r o w-b)$ and outer boundary ring. The annular fine structure of the subunits is clearly seen in the rectangular forms $(a \& c)$. (a) X 675-000. (b to e) X 900,000. 
we see a dense core of about $120 \AA$ diameter with no clearly discernible internal structure. Some of the molecules, however (Plate IIa, b) show faint indications of differentiated structures in the core. The dense core is surrounded by a semi-opaque ring (diameter about $180 \AA$, width $30 \AA$ ) featuring the outlines of 10 oval units (dimensions $30 \AA \times 45 \AA$ ) which are bounded by a dense ring (width about $15 \AA$ to $30 \AA$, outer diameter $230 \AA)$. This dense middle ring, which is clearly recognized in the different preparations, appears to be made of ten segments delimiting the ten internal units (Plate IIa, IIb, IId). Around the dense middle ring we find a well-defined clear annular zone (diameter $290 \AA$ to $300 \AA$, width $40 \AA$ ), in the center of which a fine dense concentric layer with a thickness of $8 \AA$ to $12 \AA$ is discernible (Plate II d). Adjacent to this zone is a dense outer boundary ring (diameter about $320 \AA$ ) which merges into the surrounding layer of phosphotungstate. In negatively stained preparations one regularly finds the embedded molecules surrounded by an electron-dense halo which might indicate a greater accumulation of the phosphotungstate around the molecules. The rectangular forms of the molecules measure about $350 \AA$ in a direction perpendicular to the subunit rows, and approximately $300 \AA$ parallel to the rows (Fig. 1). The regular rows have an average width of $40 \AA$, and exhibit a typical annular subunit structure (arrows Plate II a, c). The subunits have an average diameter of $40 \AA$, featuring a dense core of about $8 \AA$ to $10 \AA$ within a semi-opaque ring. In well-preserved rectangular forms it is possible to count 6 to 7 individual subunits in each of the six rows. This would give a total of about 42 subunits visible in that part of the rectangular forms of the molecules which is embedded in the phosphotungstate film.

Earlier physico-chemical experiments by van Bruggen et al. (1960) on mixtures of neutralized tungstate compounds with Helix pomatia hemocyanin demonstrated that this reagent does 
not lead to an irreversible dissociation of the molecules. These experiments are in agreement with other biochemical studies (Fernández-Morán, Oda, Blair \& Green, 1964) which indicate that phosphotungstate under the conditions used for specimen preparations in electron microscopy is a relatively mild reagent.

Aldehyde fixation of hemocyanin molecules prior to negative staining yields essentially the same type of structures as observed in unfixed preparations.

NTegative staining with "uranium-EDTA" (Plates I, III) gives the following average dimensions for the structures observed in the circular forms: dense core $=130 \AA$, semi-opaque ring = $190 \AA$, dense middle ring $=250 \AA$, clear annular zone $=340 \AA$, dense outer ring $=400 \AA$. Compared with the corresponding structures in the phosphotungstate preparations we observe, in addition to the slightly larger dimensions, the following distinctive features: the central core exhibits a series of $25 \AA$ to $30 \AA$ dense particles arranged in rosette-type or annular patterns (Plate III b to e); the constituent ten subunits stand out very clearly in ten-fold symmetrical arrangement occupying both the semi-opaque ring and the dense middle ring.

A fine dense circular layer of about $8 \AA$ is faintly visible in the middle of the clear annular zone. The demarcation Iine between the clear annular zone and the dense outer ring displays a typical serrated profile which may be related to the subunit structure of the molecules.

Positive staining. In a representative preparation of hemocyanin molecules positively stained with uranyl acetate (Plate IV) the characteristic concentric rings of the circular forms are well defined. The central regions of the circular forms $-15-$ 


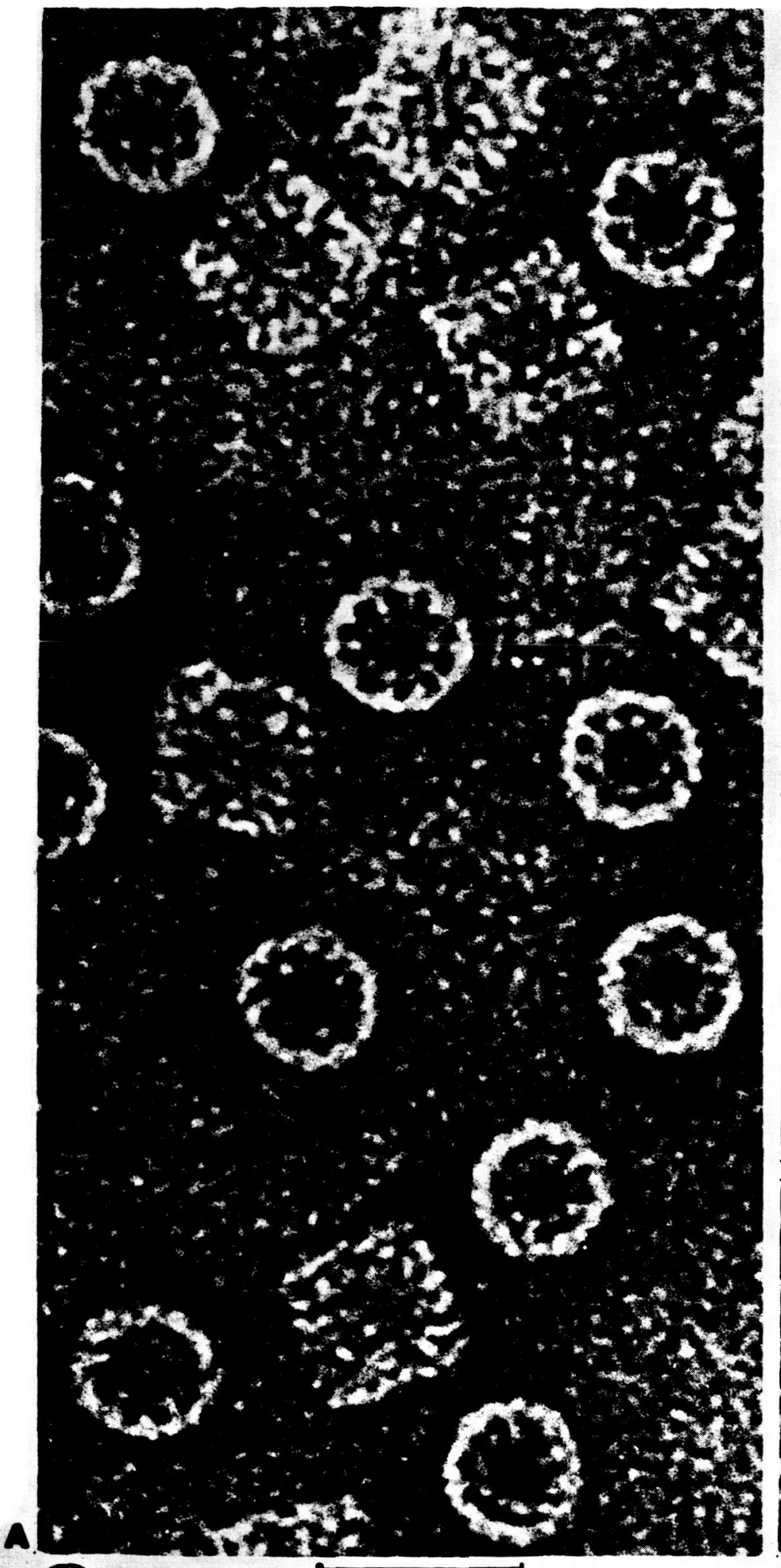

(3) PIATE 111 $500 \&$
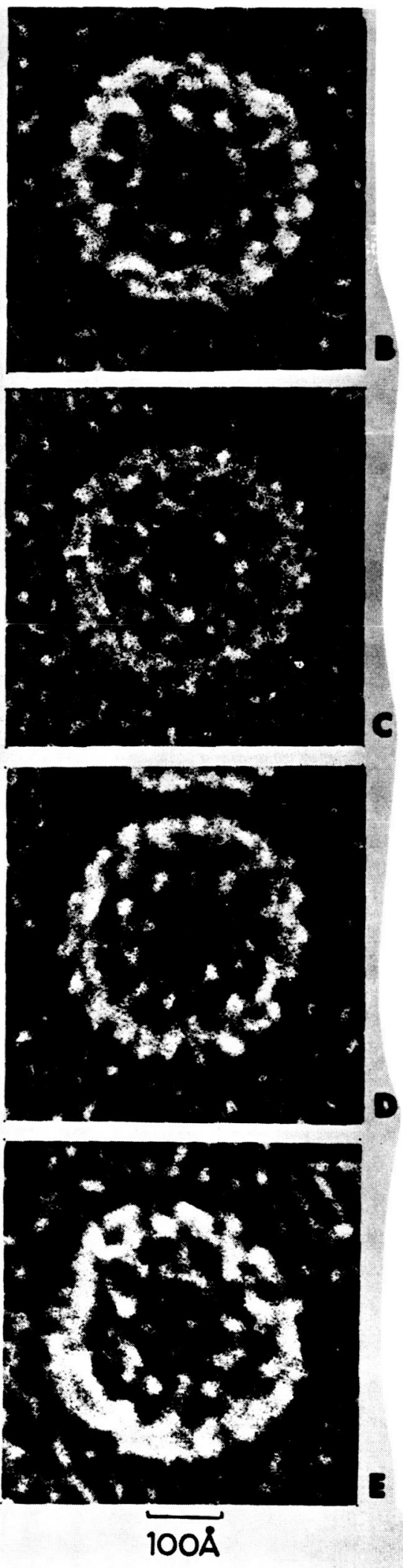

Sevaration of Helix pomatla herocyanin molecules stained with a "Uran7.-EDTA" complex without preylous fixation. The central core of the thoular forms exhibits a series of denxe particles arranged in rosette - annular patterms ( $b$ to e). The constituent ten subunits show up ary in ten-fold symmetrical arrangement occupying the region of the 14-opaque and dense middie pino. (a) X 530,000. (b to e) X 1,000,000. 

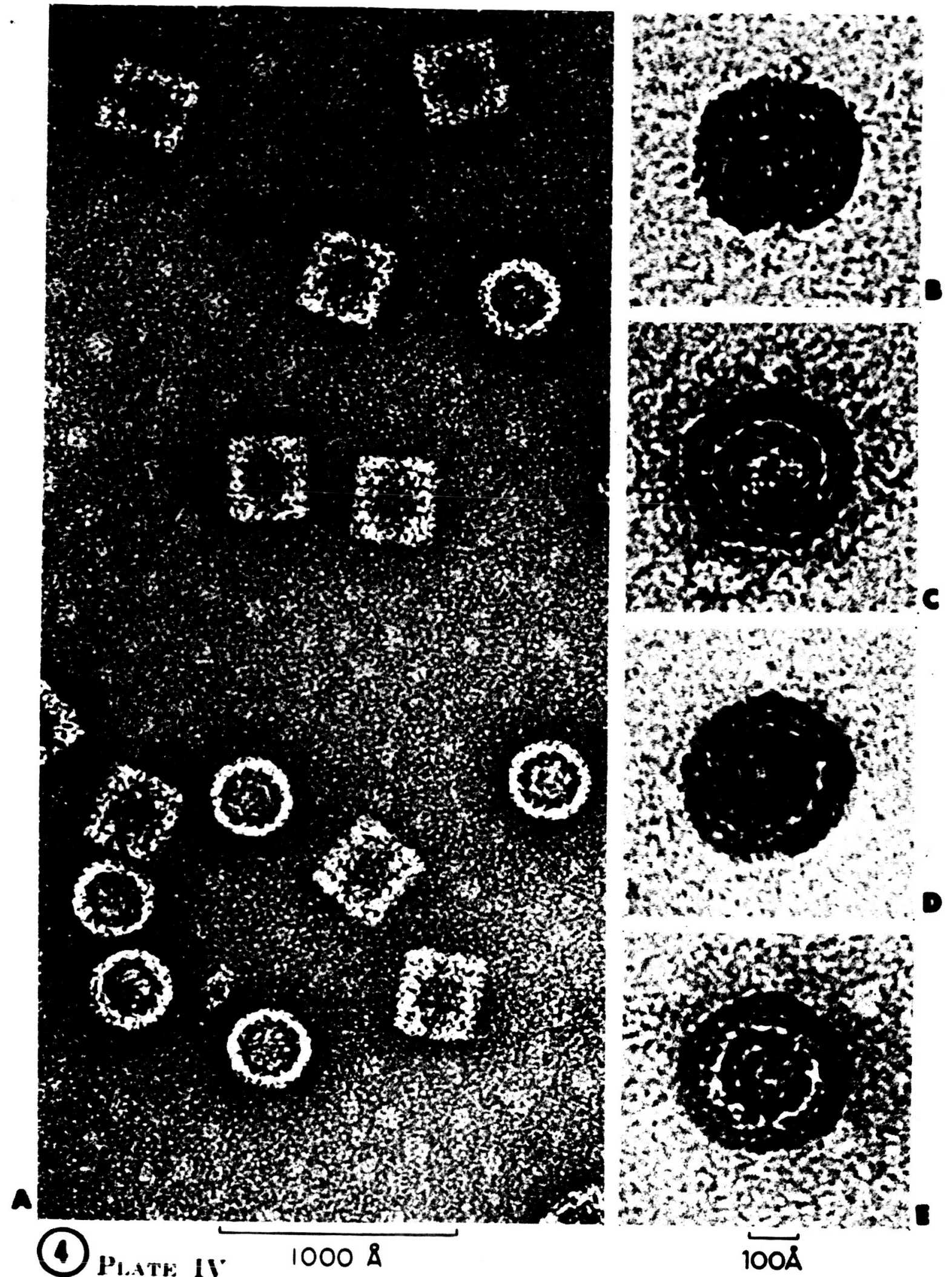

Helix pomatia hemocyanin molecules stained with uranyl acetate without previous fixation ( $b$ to $d$ ), and after fixation with glutaraldehyde (a, e). The typical concentric rings of the circular forms stand out weli, with variations in density and configuration of the central region. The dense outer ring is found closely associated with an opaque homogeneous coat ( $c$ to $e$ ), in the isolated molecules not embedded in the stain. (a) X 400,000. (b to e) X 800,000. 
of the molecules show variations in density, size and configuration. The diameter of the central region delimited by the dense middie ring varies from about $130 \AA$ in the "closed" dense forms (Plate IV d) to $170 \AA$ in the "open", semi-opaque circular forms (Plate IV c). The dense circular layer in the middle of the clear annular zone is now more pronounced (width of $15 \AA$ to $20 \AA$ ) and frequently shows a beaded substructure (Plates IV $b$ to e). The dense outer ring is found closely associated with a less dense homogeneous coat resulting in a total diameter of the order of $370 \AA$ for the separate molecules not embedded in the stain.

Interesting observations can be made when the hemocyanin molecules are adsorbed on extremely thin bentonite or montmorillonite lamellae ( $10 \AA$ to $30 \AA$ thick) prepared according to the method of McLaren and Peterson (1961). In these preparations the hemocyanin molecules are sandwiched between the underlying carbon film and the thin bentonite lamellae. Upon drying, a characteristic spreading or compression effect is noted which displaces the dense central core eccentrically and flattens out the less-dense subunit structures of the outer layers. Under favorable conditions it is also possible to examine these sandwiched preparations with a liquid nitrogen cold stage at low temperatures, thus partially preserving them in their hydrated state. Although under these conditions the molecules are exceedingly sensitive to electron beam radiation effects and rapid etching sets in, their general dimensions appear to be about $20 \%$ larger than those of the standard dried negatively stained preparations.

\section{Fixed and Carbon-Coated Preparations.}

Hemocyanin molecules fixed with $1.5 \%$ buffered glutaraldehyde or with $1 \%$ to $3 \%$ neutralized formaldehyde prior to negative or positive staining show essentially the same structures as the unfixed molecules. However, the dense outer coat stands 
out more clearly. The coat can be stabilized by vertical deposition of a very thin carbon film in an ultrahigh vacuum.

In order to gain a better understanding of the three-dimensional configuration of the hemocyanin molecules, specimens fixed with aldehydes were obliquely shadowed with carbon in an ultrahigh vacuum $\left(10^{-8}\right.$ to $\left.10^{-9} \mathrm{~mm} \mathrm{Hg}\right)$. The enhanced contrast obtained with the highly coherent microbeam illumination makes the faint carbon shadows clearly visible (Plates $\mathrm{V}, \mathrm{VI})$. The individual hemocyanin molecules stand out as cylindrical or barrel-shaped structures projecting sharp shadows. The outer coat surrounding the molecules is slightly eccentric and broadened in the direction of the carbon source. It exhibits a regular series of concentric rings of the order of $10 \AA$ to $20 \AA$ (Plate $V$ a to e).

Approximately six rings can be counted with a gap between the third and the fourth ring. The rings show a regular beaded structure of about $10 \AA$ to $20 \AA$. Although the possibility may be considered that the concentric rings result from successive depositions of carbon during repeated shadowing, it is more probable that they correspond to an actual structure. This conclusion is based on examination of a large number of plates in which shadowing was performed from different angles and under varying conditions, without appreciably modifying the number and fine structure of the concentric rings of the outer coat. In these shadowed preparations the subunit fine structure of the rectangular forms is also particularly well defined. As shown in Plate $V c$, a characteristic profile of the shadows with two peaks can be seen. These peaks are probably indicative of a groove parallel to the rows of subunits corresponding to the middle of the molecule where dissociation usually occurs. Another type of groove, perpendicular to the direction of the rows, was described by van Bruggen et al.(1962a). 


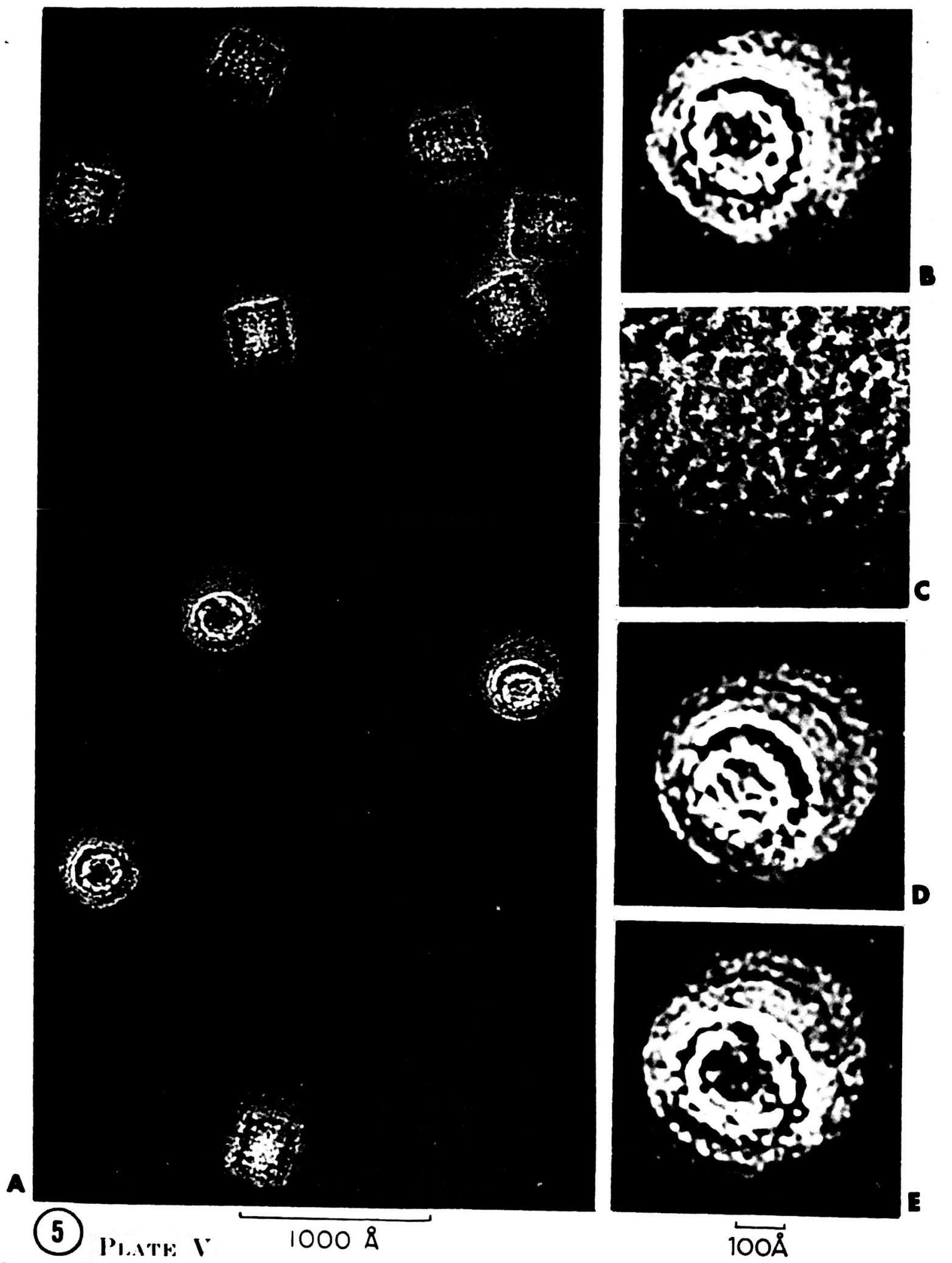

Helix pomatia hemocyanin molecules fixed with glutaraldehyde, stained with uranyl acetate and shadowed with carbon in an ultrahigh vacuum. As a result of the enhanced contrast obtained with coherent microbeam 1IIumination, the cylindrical hemocyanin molecules stand out distinctly, projecting sharp shadows (a). The slightly eccentric outer coat exhibits a series of concentric rings with a regular beaded structure (b,d,e). The rectangular forms project characteristic shadows with two peaks (c) which may correspond to a central groove parallel to the rows of subunits. Reversed-contrast print. (a) X 320,000. (b to e) X 800,000. 

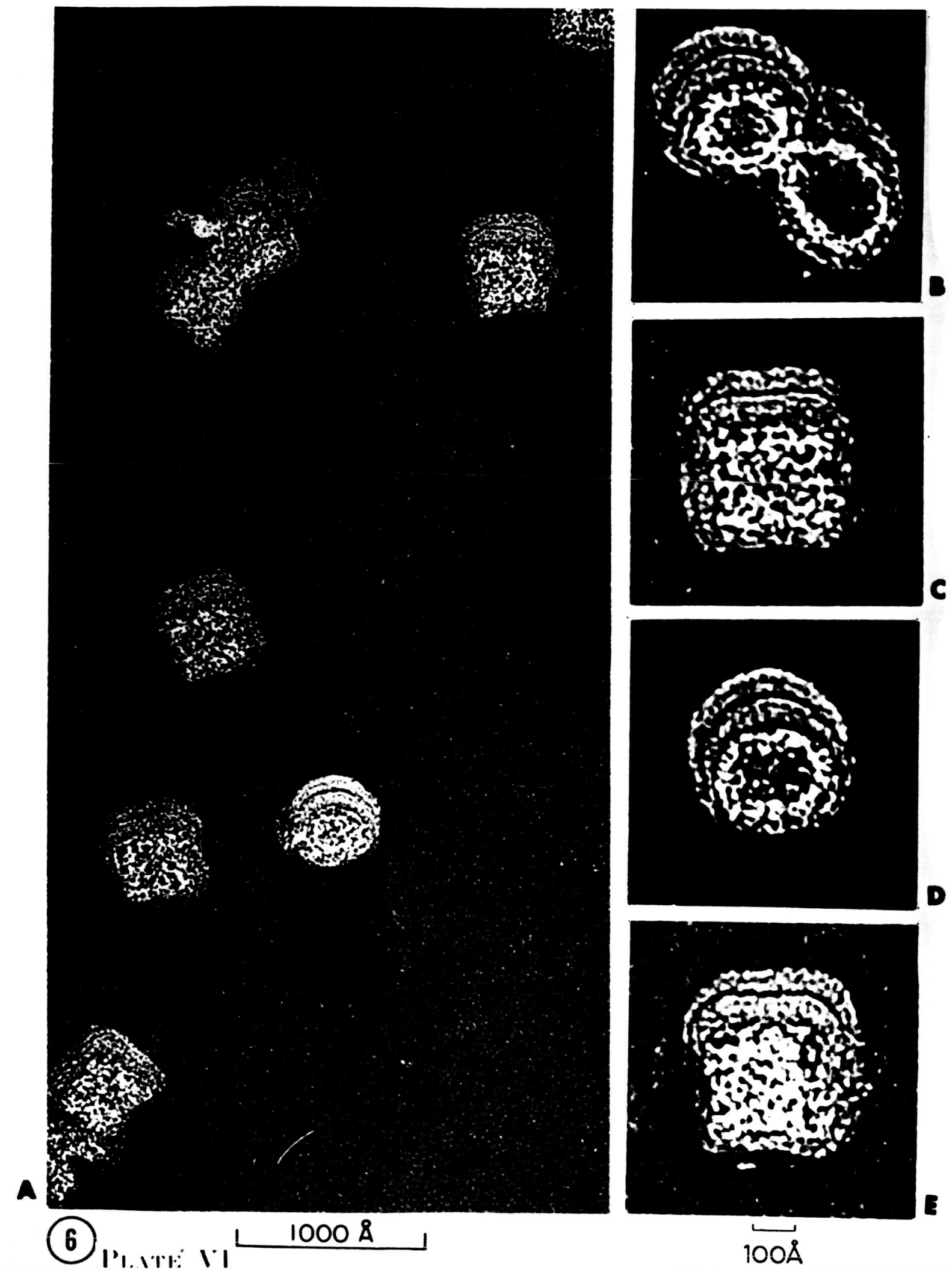

Helix pomatia apohemocyanin molecules fixed with glutaraldehyde, stained with uranyl acetate and shadowed with carbon in an ultrahigh vacuum. The three-dimensional appearance and fine structure is similar to the corresponding hemocyanin preparations. The outer coat is conspicuous in both the circular and rectangular forms, and the individual molecules appear to be slightly larger and more permeable to stains. Reversed-contrast print. (a) X 320,000. (b to e) X 700,000. 
(ii) Apohemocyanin. Examination of a wide variety of negatively and positively stained fixed and unfixed apecimens of apohemocyanin failed to show significant differences with respect to the structure of hemocyanin. This is in agreement with the preliminary findings reported by van Bruggen et al. (1962a)

The only differences detected were found in uranyl-stained and shadowed preparations (Plate VI), and in phosphotungstate cross-sprayed specimens (Plate VII). In these preparations the inner part of the molecule seems to be more opened up and permeable to the stain. This results in more marked differences in the configuration of the central regions of the circular forms. The dimensions of the apohemocyanin molecules appear to be slightly larger (about 10\% to 15\%) than those of the hemocyanin molecules; the outer coat is also more conspicuous.

Certain reproducible differences could be detected in a series of controlled etching experiments using microbeam illumination in combination with the cold stage. Under certain conditions (increased intensity of microbeam illumination, presence of water vapor and residual gases, etc.) a characteristic etching effect can be observed in negatively stained specimens which have been sandwiched between carbon films. As shown in Plates VII $a, b$, one can observe directly a partial etching away of certain parts of the hemocyanin and apohemoyanin molecules. In hemocyanin molecules (Plate VII a) the clear annular zone adjacent to the middle dense ring is gradually etched away. However, some of the details of the middle dense ring and central core are retained. By contrast, in the apohemocyanin molecules (Plate VII b) there is extensive obliteration of fine detail in the outer and intermediate zones with partial preservation of the dense central regions. However, interpretation of the results of these etching experiments is still uncertain, and further work is necessary. 


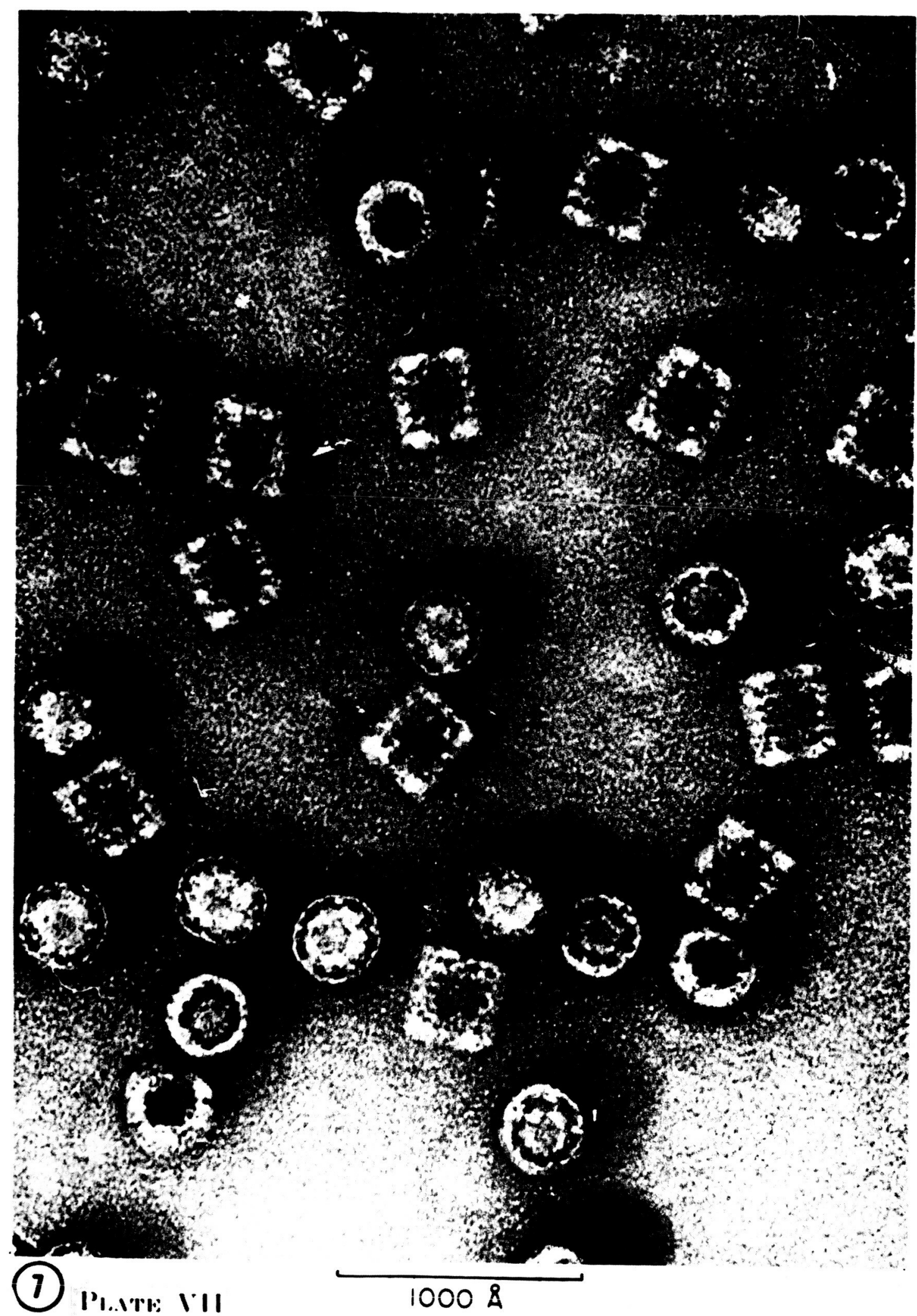

Preparation of Helix pomatia apohemocyanin molecules negatively stained with sodium phosphotungstate by the double-spray method without previous fixation. The marked differences in the density and configuration of the central regions of the circular forms may result from enhanced permeability of the apohemocyanin molecules to the stain. X 400,000. 

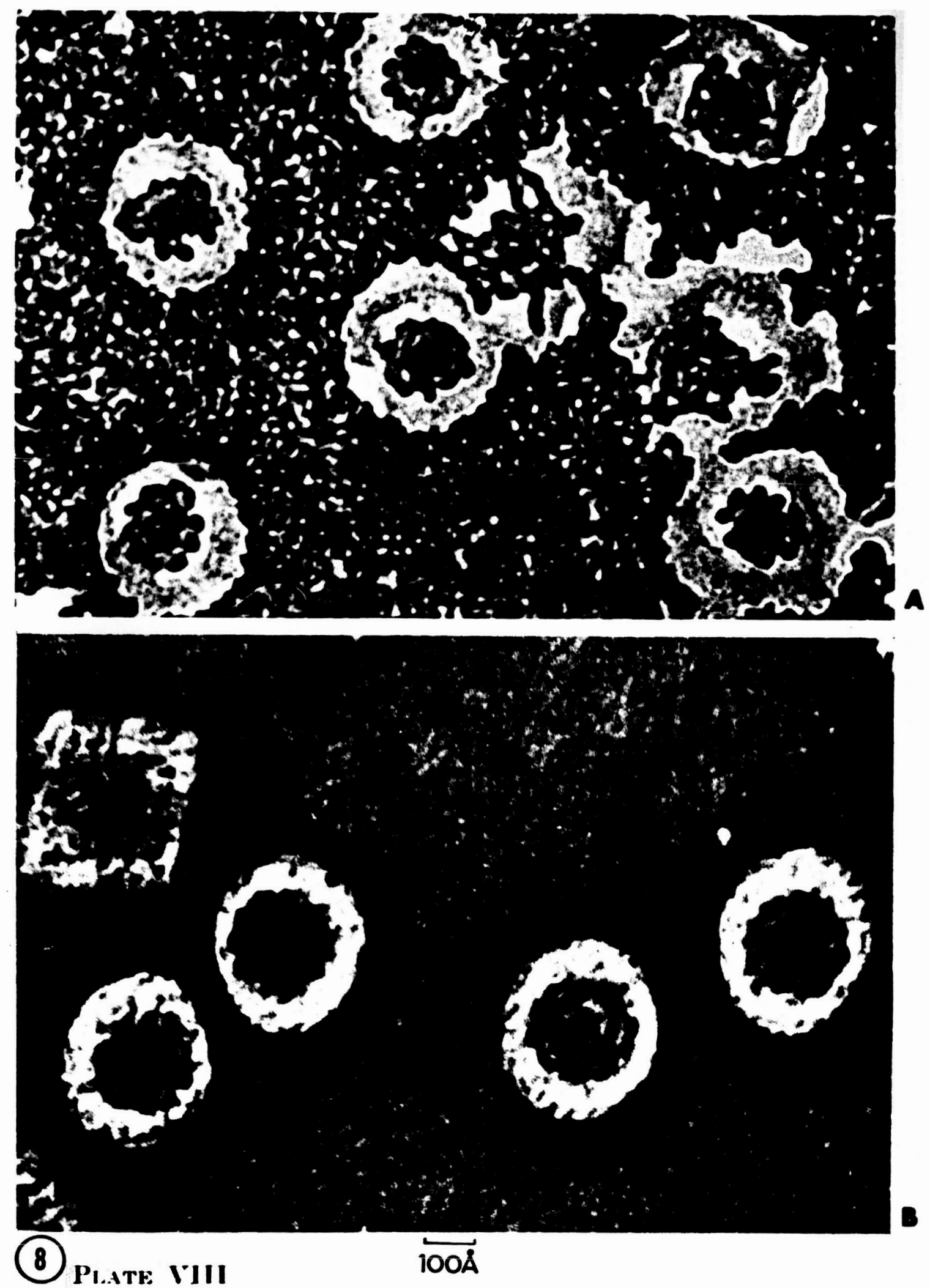

Electron micrographs of Helix pomatia hemocyanin (a) and apohemocyanin molecules (b) which have been negatively stained, sandwiched between carbon films, and subjected to controlled etching with microbeam 1llumination at low temperatures. Selective etching away of certain parts of these molecules can be observed which may possibly be related to the modifications induced by the treatment for copper removal. (a) $X 780,000$ and (b) X 800,000. 
(iii) Busycon canaliculatum hemocyanin

Busycon hemocyanin molecules show, in negatively and positively stained preparations (Plates IX, X), circular and 6row rectangular structures which are essentially similar to those of Helix pomatia hemocyanin.

More elongated rectangles having the same width show up with 9 rows (length $520 \AA$ ) and 12 rows (length $680 \AA$ ) (Plates IX, $X)$. Closer examination reveals an irregularity in the spacing at row 6 and 7 for the 9-row structures, and two irregularities respectively at row 3 and 4 and at row 6 and 7 for the 12-row rectangles.

\section{(iv) Busycon apohemocyanin}

Busycon apohemocyanin and hemocyanin molecules showed the same type of minor differences (Plate XI a, b) as described for the corresponding preparations of Helix pomatia.

As shown in Plates XII and XIII, certain typical modifications of the internal core of the circular forms were consistently found in fixed and uranyl acetate stained preparations. Instead of the circular rows of particles in the inner core, one finds large numbers of molecules with rodlike or elongated dense structures (Plate XIII). The rods are about $50 \AA$ to $100 \AA$ long, and approximately $20 \AA$ to $40 \AA$ wide. In addition to these modifications there appears to be a general swelling of the whole molecule. The outer coat is very marked and has an average width of about $50 \AA$ to $80 \AA$. In addition to the clearly visible structures there appears to be a general matrix-like material which is extremely fine grained, permeating the entire molecular structure. 

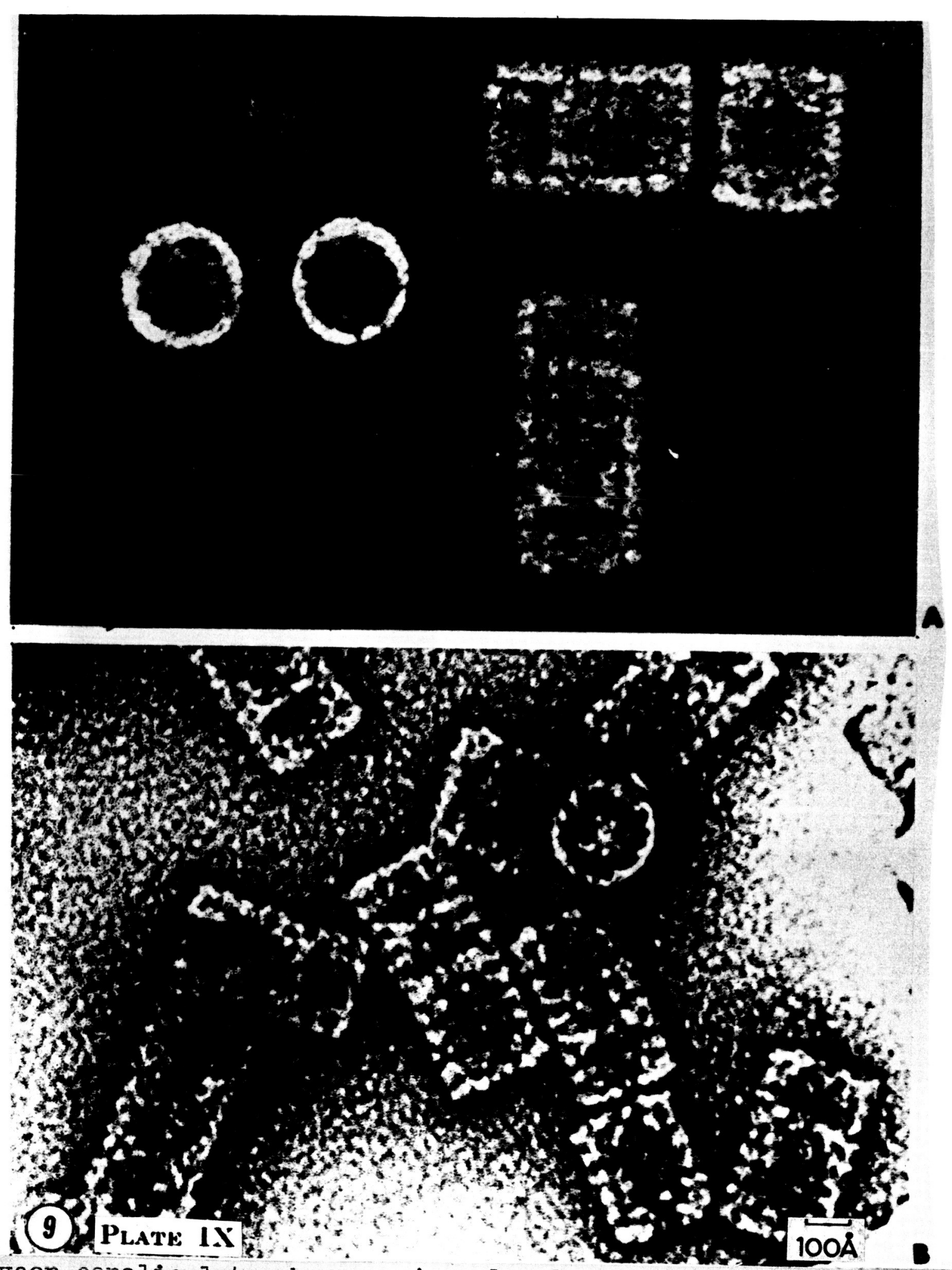

(a) Busycon canaliculatum hemocyanin molecules negatively stalned with potassium phosphotungstate without prior fixation. The circular forms closely resemble those of Helix pomatia hemocyanin. Typical elongated rectangular forms are visible with nine rows (520A long) and 12 rows (680A long). X 720,000. (b) Busycon canaliculatum hemocyanin molecules stained with uranyl acetate without prior fixation. These molecules are embedded in a thin film of stain extending over the holes of a fenestrated film, distinctiy showing the irregularity in the spacing at row 6 and 7 for the 9-row rectangular structures, and the irregularities around row 3 and 6 for the 12-row structures. X 700,000 . 


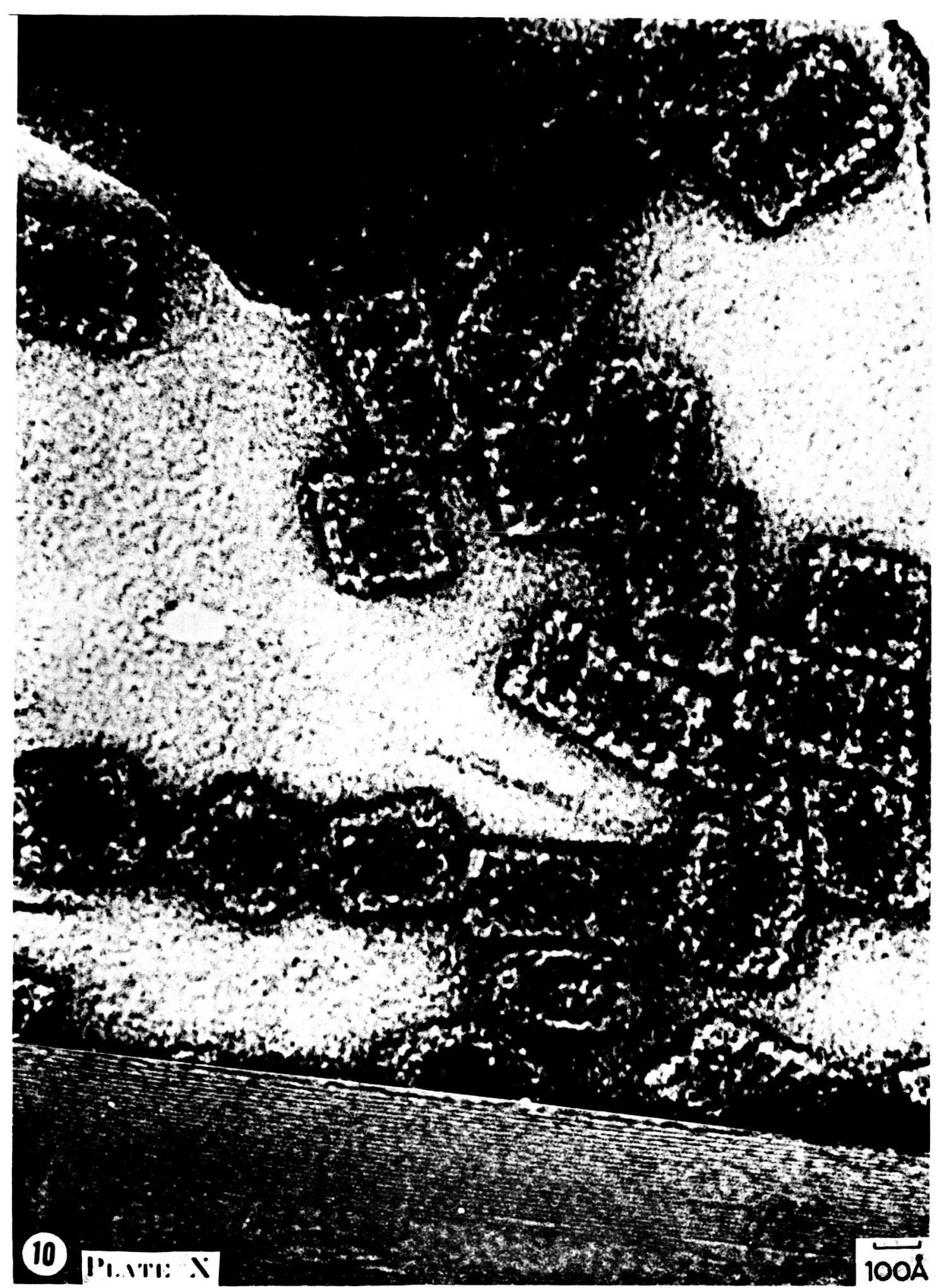

Preparation of Busycon canaliculatum hemocyanin molecules stained and embedded in a thin film of uranyl acetate extending over the holes of a fenestrated carbon film reinforced with ultrathin asbestos filaments (I00A diameter). Numerous molecules are found adhering to the slender asbestos filaments in different orientations, while others are supported within extremely thin films of the negative stain bridging the resulting network of submicroscopic gaps. The individual subunits are seen in parallel array in the 6-, 9-, and 12-row rectangular forms featuring a dense serrated border. The asbestos filaments (shown in bottom insert at $X$ 1,000,000) exhibit a characteristic crystalline lattice period of $7.3 \AA$ which serves as an accurate calibration standard. X 720,000. 

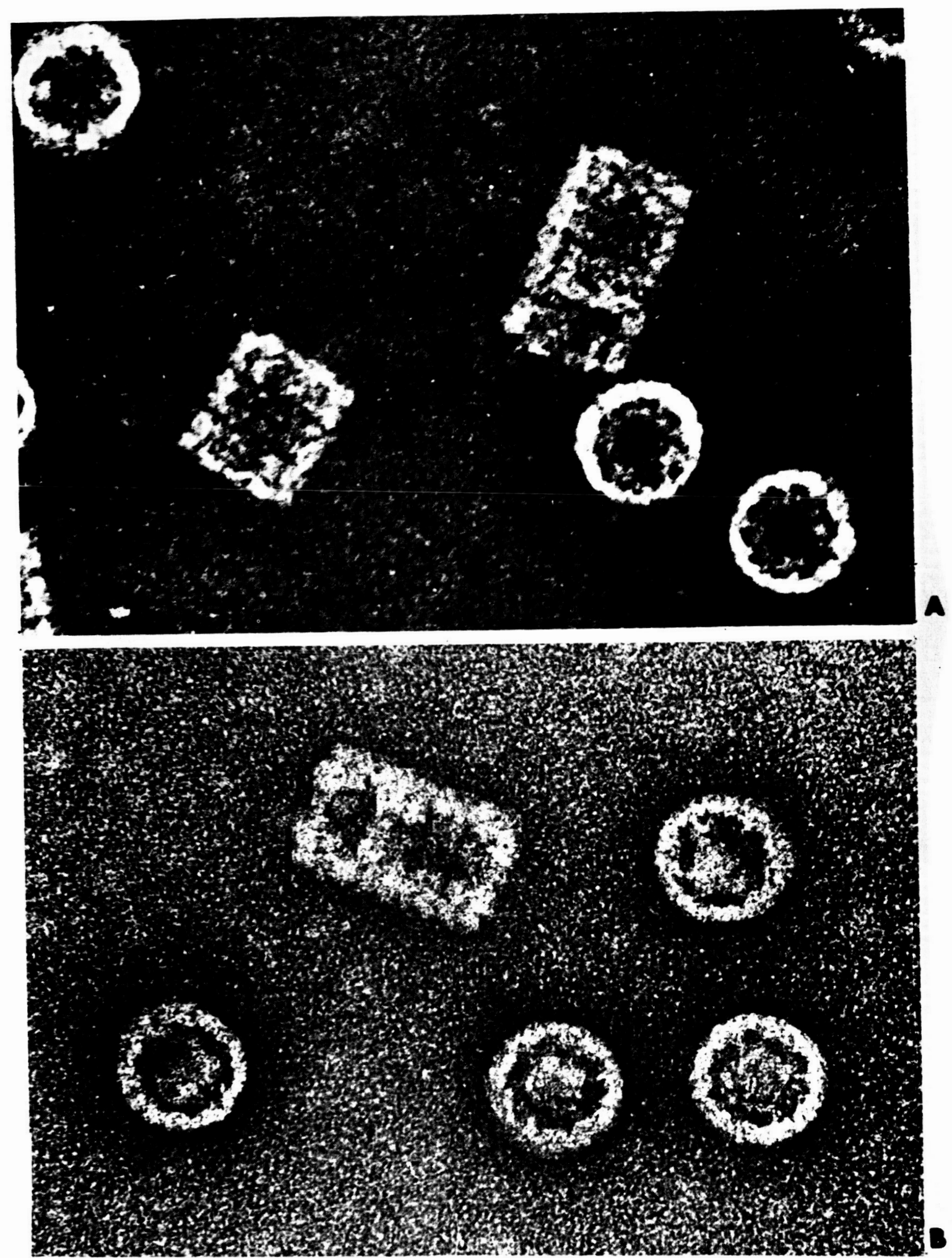

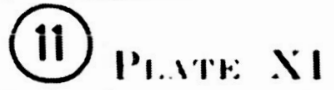

$500 \&$

Electron micrographs of Busycon canaliculatum hemocyanin (a) and apohemocyanin (b) molecules negatively stained, showing the same type of minor stmuctural differences mainly in the central region of the circular forms as observed in the corresponding preparations of Helix pomatia. X 670,000 . 

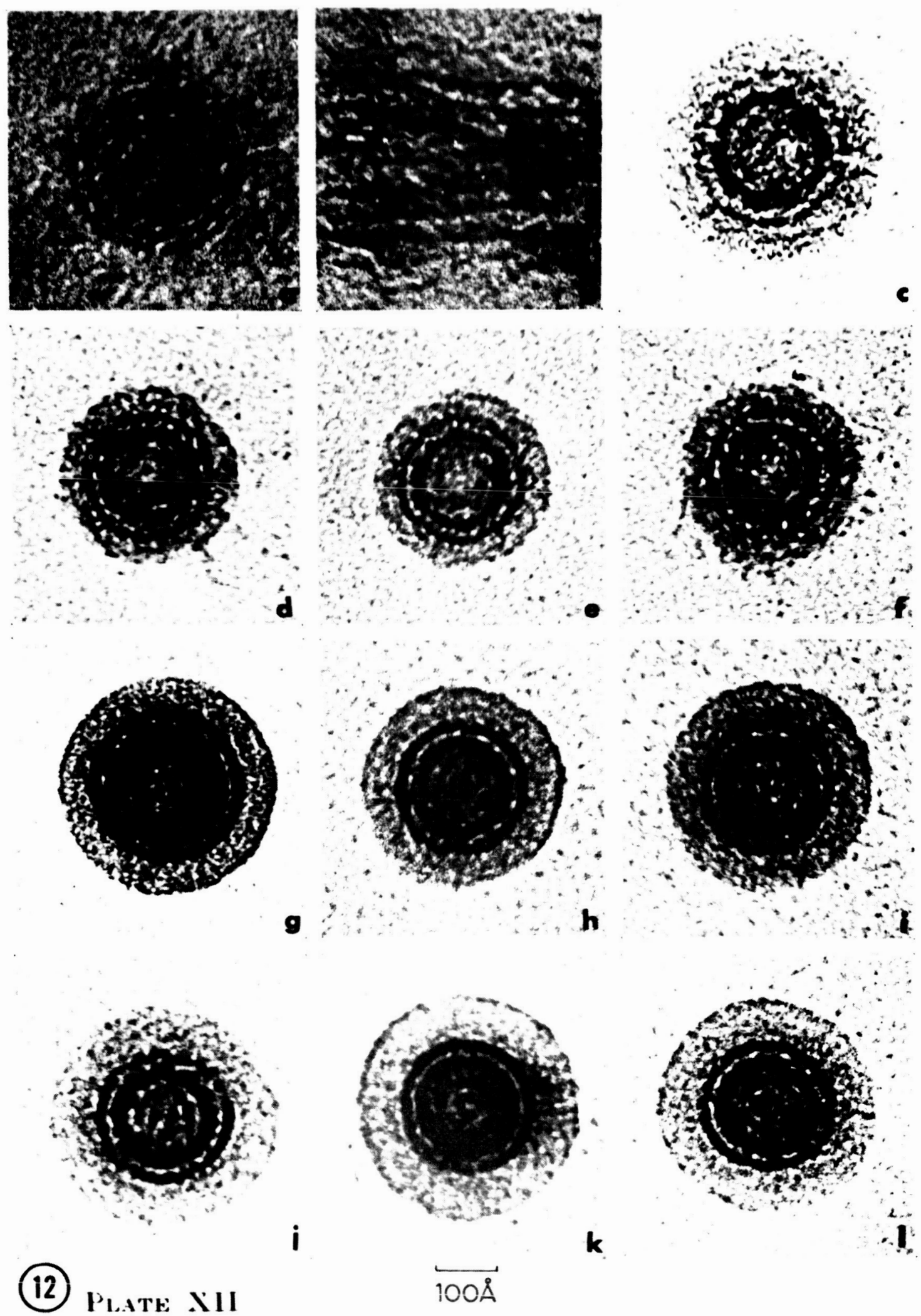

Electron micrographs of representative hemocyanin ( a,b,c) and apohemocyanin (d-l) molecules of Busycon canaliculatum stained with uranyl acetate without prior fixation $(a-f)$, and after fixation with glutaraldehyde $(g-1)$. Characteristic stmetural modifications are observed in the dense core of the circular forms, and the outer coat is generalIy broadened and wel1-defined $(g-1) \cdot(a-1)$ X 880,000.(g-1) X 1, 000,000 . 

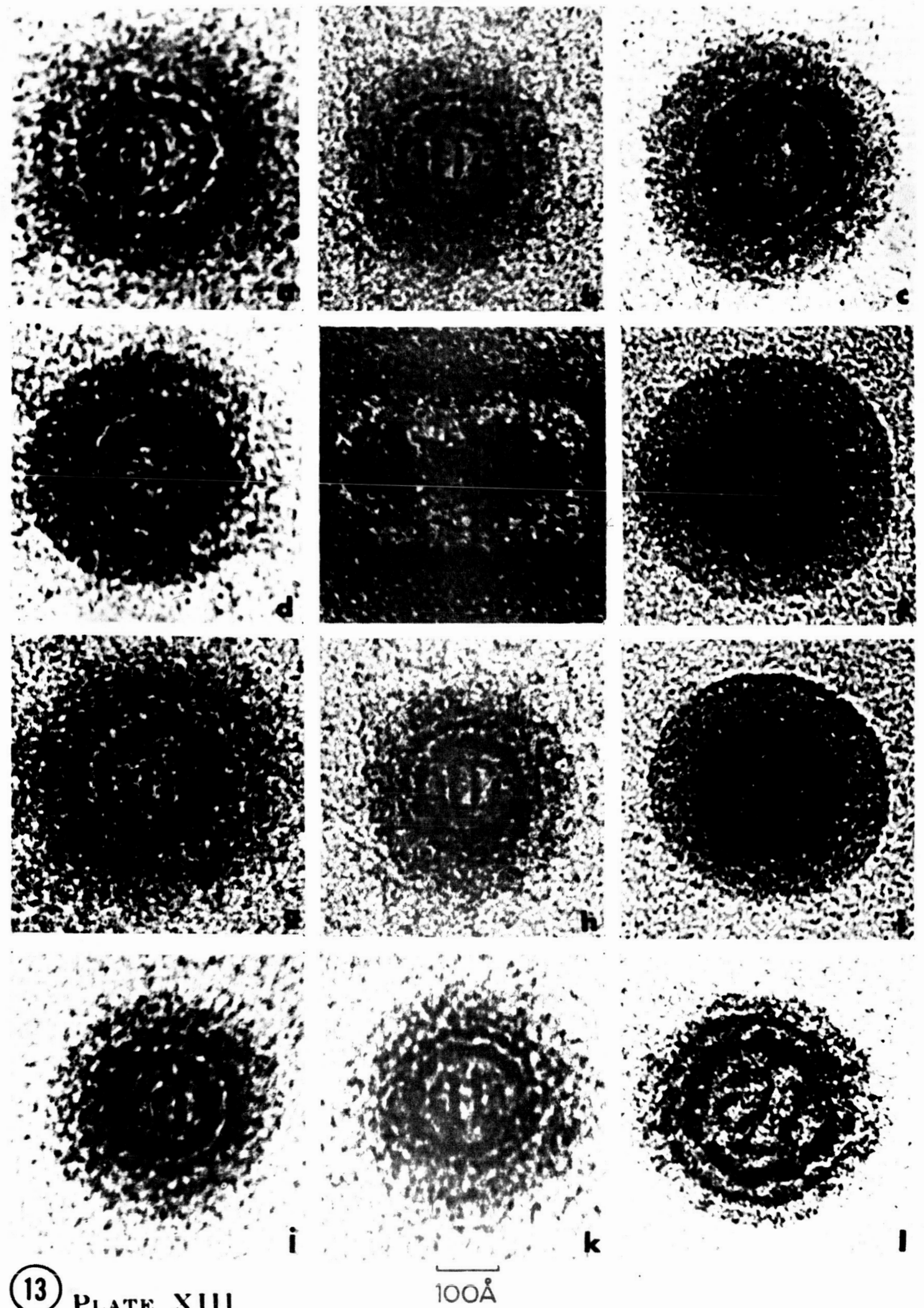

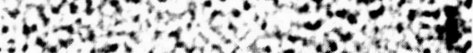

\section{(13)}

PIATE XIII

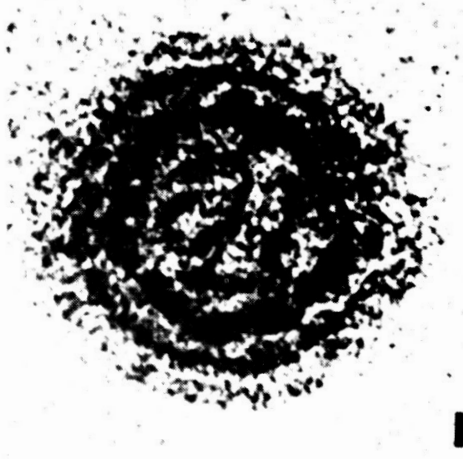

Electron micrographs of representative apohemocyanin molecules of Busycon canaliculatum stained with uranyl acetate after fixation with glutaraldehyde. Numerous molecules are found with rod-like or elongated dense structures in the central region of the circular forms, with a variety of intermediate configurations. The outer coat is very marked $(c, f, i)$ and has an average width of $50 \AA$ to $80 \AA$. There also appears to. be a general swelling of the whole molecule. X I,000,000. 


\section{(v) Loligo pealei}

Electron micrographs of glutaraldehyde fixed molecules from Loligo pealei hemocyanin negatively stained with potassium phosphotungstate (Plate XIV) confirm essentially the previous findings reported by van Bruggen et $a 1$. (1962b) for another Decapod Sepia officinalis.

The molecules appear as circles with a ten-fold symmetry and a diameter of about $330 \AA$ and as rectangles of $370 \AA$ $\mathrm{x} 140 \AA$. Details in the molecules are obscured, which might be due to the fixation procedure.

Staining with uranyl acetate (Plate XVI) shows much more details, but unfortunately it is very difficult to visualize the rectangles in this way. Spraying of a mixture of the hemocyanin and "uranium-EDTA" gave good structural preservation. However, now the background grain from the stain was too coarse. Floating of a specimen thus prepared in uranyl acetate for about 15 seconds gives the result shown in Plate XV. Both circles and squares show very fine structural details. It is difficult to see the separate subunits in the outer ring of the circles. The inner ring clearly consists of 10 subunits. The diameter of these subunits is $20 \AA$ to $30 \AA$, and the distance between them is $40 \AA$ to $50 \AA$. In contrast to Plate XIV stained structures are observed in the core of the inner ring of the circles in Plate XV. These structures are less clear than those seen in Helix and Busycon hemocyanin. The rectangles show the subunits in 3 rows parallel to the longest side. The subunits seem to be arrayed in a close-packed staggered tern in contrast to Helix and Busycon hemocyanin, where the subunits from different rows seem to stand exactly above each other. Further, the rectangles from Loligo appear to stain completely symmetrically, while the corresponding structures of Helix and Busycon show different densities for the first and the last rows. In addition to the circles and rectangles, smaller components are observed that do not show a characteristic structure. 

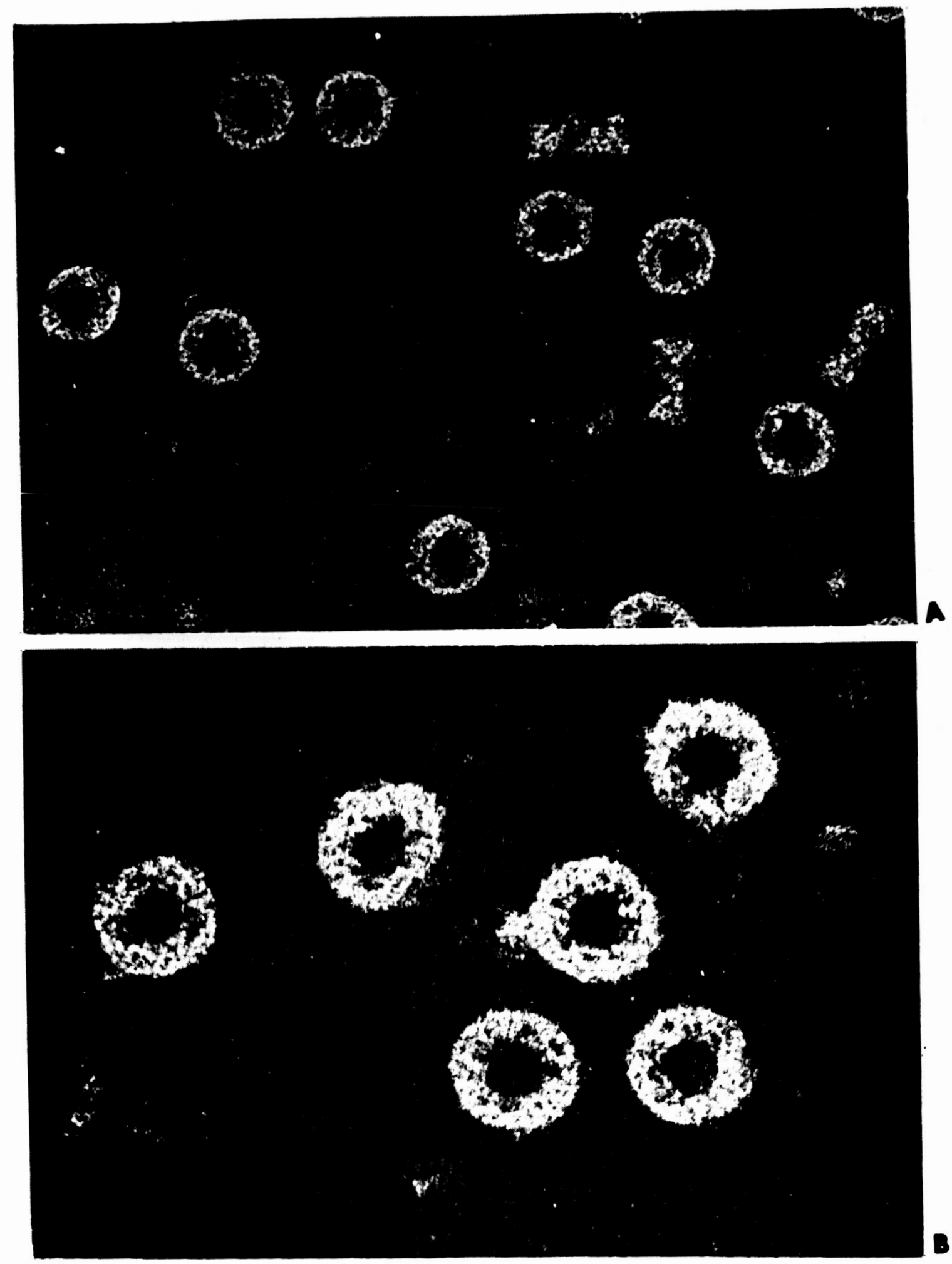

(14) Plate XIV

$1000 \AA$

Loligo pealei hemocyanin molecules glutaraldehyde fixed and negatively stained with potasium phosphotungstate. The molecules appear as circles (diameter about $330 \AA$ ) and as rectangles of about $140 \AA \mathrm{X} 370 \AA$. Subunit structure of circular and rectangular forms is discernible. (a) X 400,000. (b) X 600,000. 


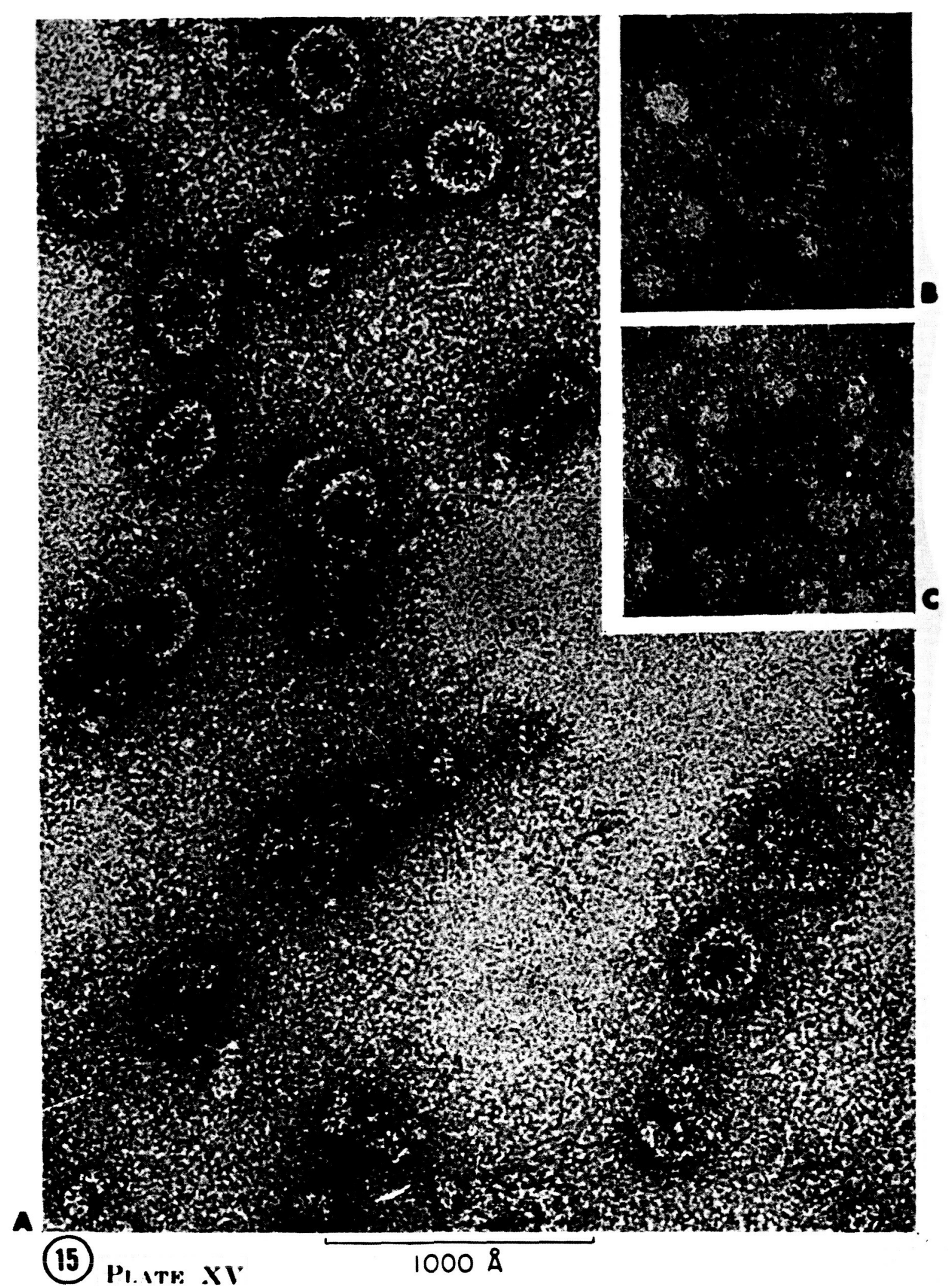

(a) to (c) Loligo pealei hemocyanin molecules sprayed as a mixture with with "uranium-EDTA and then floated on uranyl acetate for about 15 seconds. The molecules appear as circles (diameter about $310 \AA$ ) and as rectangles of about $160 \AA$ x $355 \AA$. The circular forms show subunit structure with ten-fold symmetry; the rectangular forms show 3 rows of subunits in a close-packed staggered pattern. Smaller components (diameter $70 \AA$ to $100 \AA$ ) bullt from 3 to 5 subunits are also visible. (a) $\times 450,000$, (b) $\mathrm{x} 675,000$. 


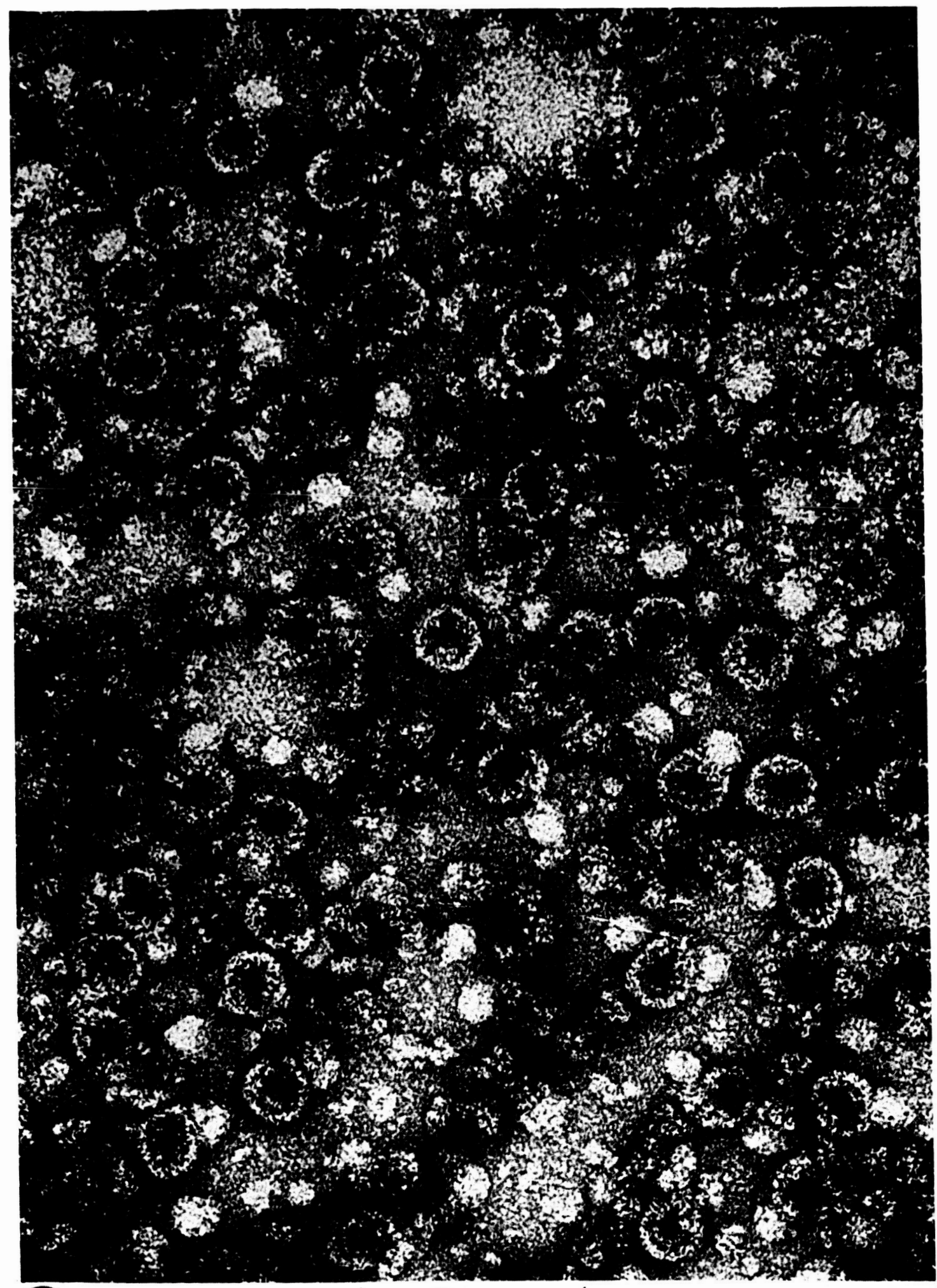

\section{(16) Plate XVI}

$1000 \AA$

Loligo pealei hemocyanin molecules negatively stained with uranyl acetate. The dimensions, symmetry and substructure of the molecules are the same as described for Plate XV. Note the fine details visible in the subunits. $\mathrm{x} 360,000$. 


\section{(vi) Loligo apohemocyanin}

Specimens of glutaraldehyde-fixed Loligo apohemocyanin, which were negatively stained with phosphotungstate, do not show any reproducible differences of significance as compared with the hemocyanin. However, it was noticed that in many cases the inner core appears to be more dense. Most of the structures here were broken up into segments. In some of the areas examined at high magnifications there were indications that not all of the subunits exhibited the characteristic dense, internal core.

Loligo apohemocyanin, prepared according to the method of Cohen \& Van Holde (1964) sprayed as a mixture with "uraniumEDTA", followed by floating on uranyl acetate (Plate XVII) shows circles and rectangles that are similar to those of the hemocyanin prepared in the same way. A larger amount of smaller components is observed. The smallest components with a diameter of $70 \AA$ to $100 \AA$ are built from about 3 subunits, while the larger ones are formed by a combination of two or more of these structures. Dimers measuring $70 \AA$ to $100 \AA \times 230 \AA$ to $270 \AA$ occur frequently.

\section{(vii) Limulus polyphemus}

Depending on the composition of the medium, Limulus polyphemus hemocyanin contains components with sedimentation constants of $6 \mathrm{~s}, 16 \mathrm{~s}, 24 \mathrm{~s}, 35 \mathrm{~s}$, and $57 \mathrm{~s}$.

Confirming the results reported previously by van Bruggen (1964) the $57 \mathrm{~s}$ component, negatively stained with sodium phosphotungstate at $\mathrm{pH} 7$ (Plate XVIII) shows up as circles with a diameter of about $240 \AA$, or as rectangles with dimensions of $210 \AA \times 240 \AA$ composed of two parallel rows, each measuring about $90 \AA \times 240 \AA$. The $35 \mathrm{~s}$ component (Plates XVIII to XX) appears as a square consisting of 4 smaller squares connected at one of their corners as described first by Levin (1963c). 

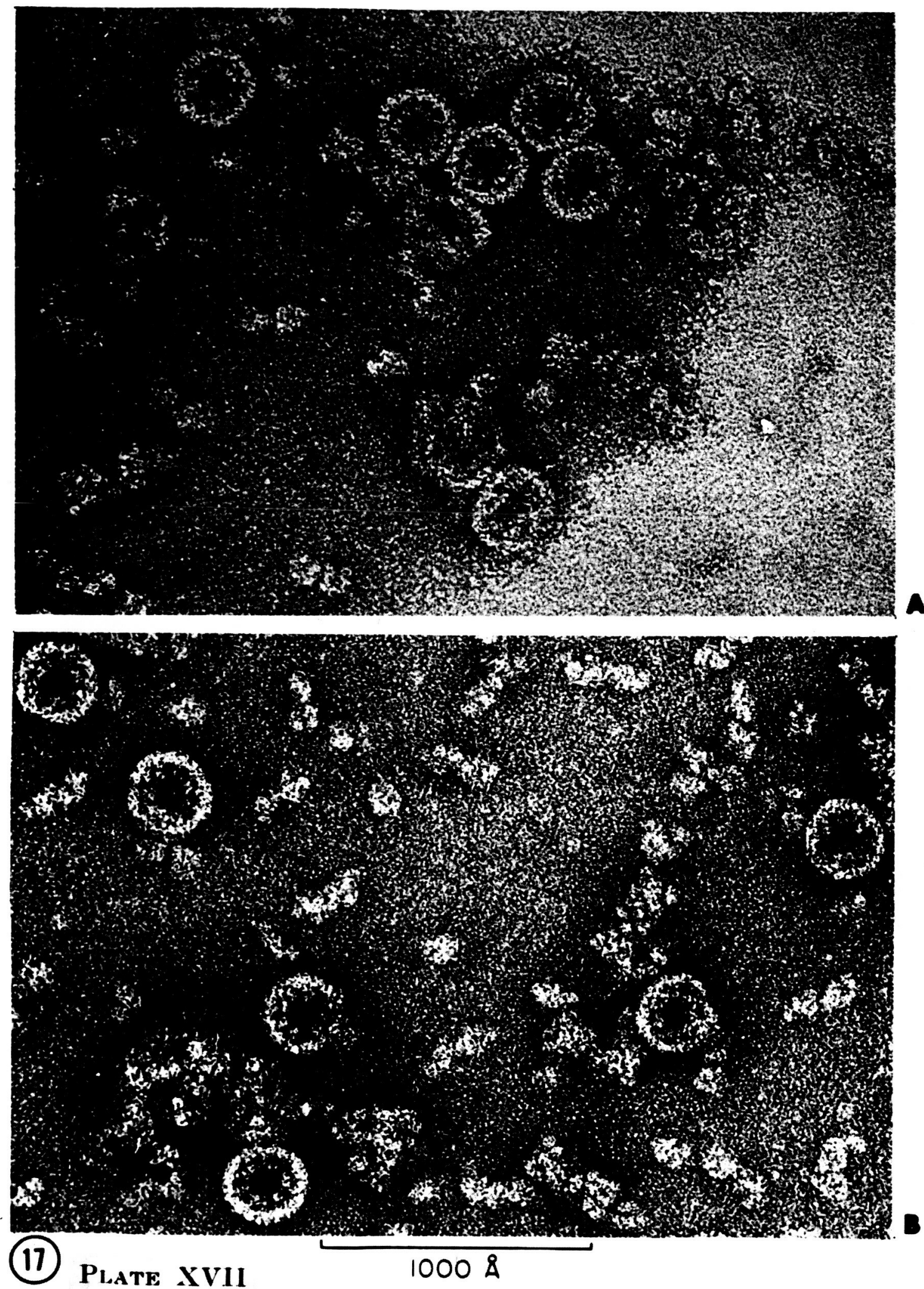

Iollgo pealla apohemocyanin molecules sprayed as a mixture with "uraniumIDIF and then floated on uranyl acetate for about 15 seconds. The mole-

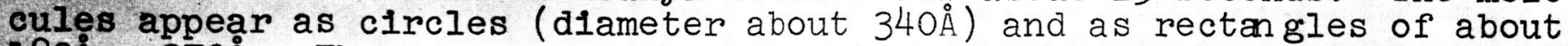
$180 \AA \times 370 \AA$. The symmetry and substructure of the molecules is the same as described for Loligo hemocyanin. Many smaller components are observed. The smallest with a diameter of $70 \AA$ to $100 \AA$ are built from 3 to 5 subunits, while the larger ones are formed by a combination of two or more of these structures. (a) and (b) X 450,000. 


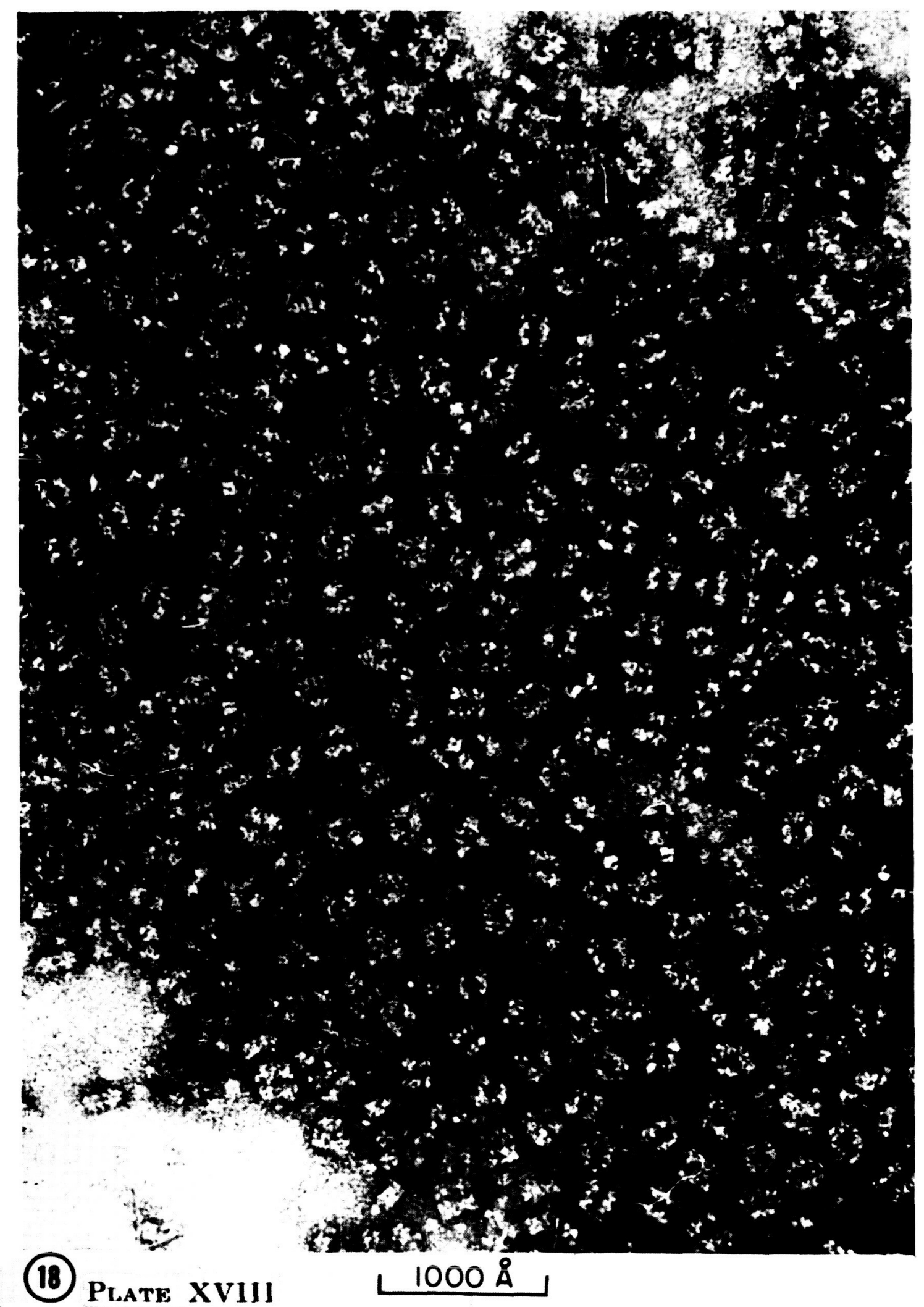

Itmulus polyphemus hemocyanin molecules negatively stained with sodium phosphotungstate. The $16 \mathrm{~s}$ component is observed as a small square or rectangle (sides $80 \AA$ to $100 \AA$ ) or hexagon (diameter $120 \AA$ to $150 \AA$ ). The $24 \mathrm{~s}$ component, especially seen at the border region where the molecules are dissociating, is often observed as a combination of a hexagon with a square or rectangle: The 35s component appears as a tetramer of 4 squares (diameter about $240 \AA$ ) or as rectangles with dimensions of $210 \AA \times 240 \AA$, composed of 2 parallel rows, each measuring about $90 \AA \times 240 \AA . \quad x 285,000$. 

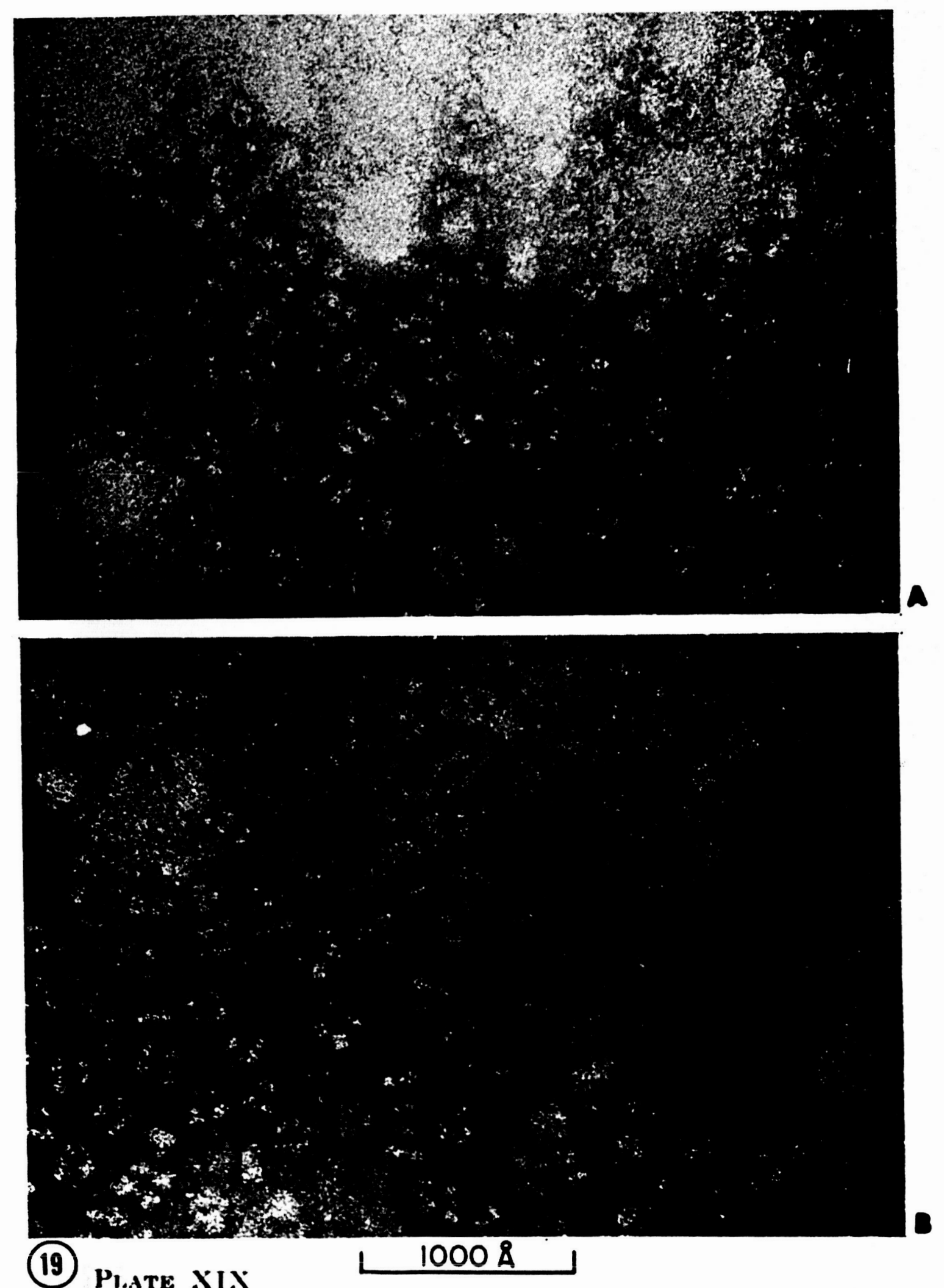

Iimulus polyphemus hemocyanin molecules negatively stained with potassium phosphotungstate. The dimensions, symmetry, and substructure of the molecules is the same as described for Plate XVIII. Note expecialiy in (a) a ring structure with a diameter of about $100 \AA$, probably corresponding to another protein present in Iimulus hemolymph. The elongated.structure of $100 \AA$ x $450 \AA$ with a spacing of about $65 \AA$ probably is a side view of a stack of several of these $100 \AA$ rings. X 360,000 . 

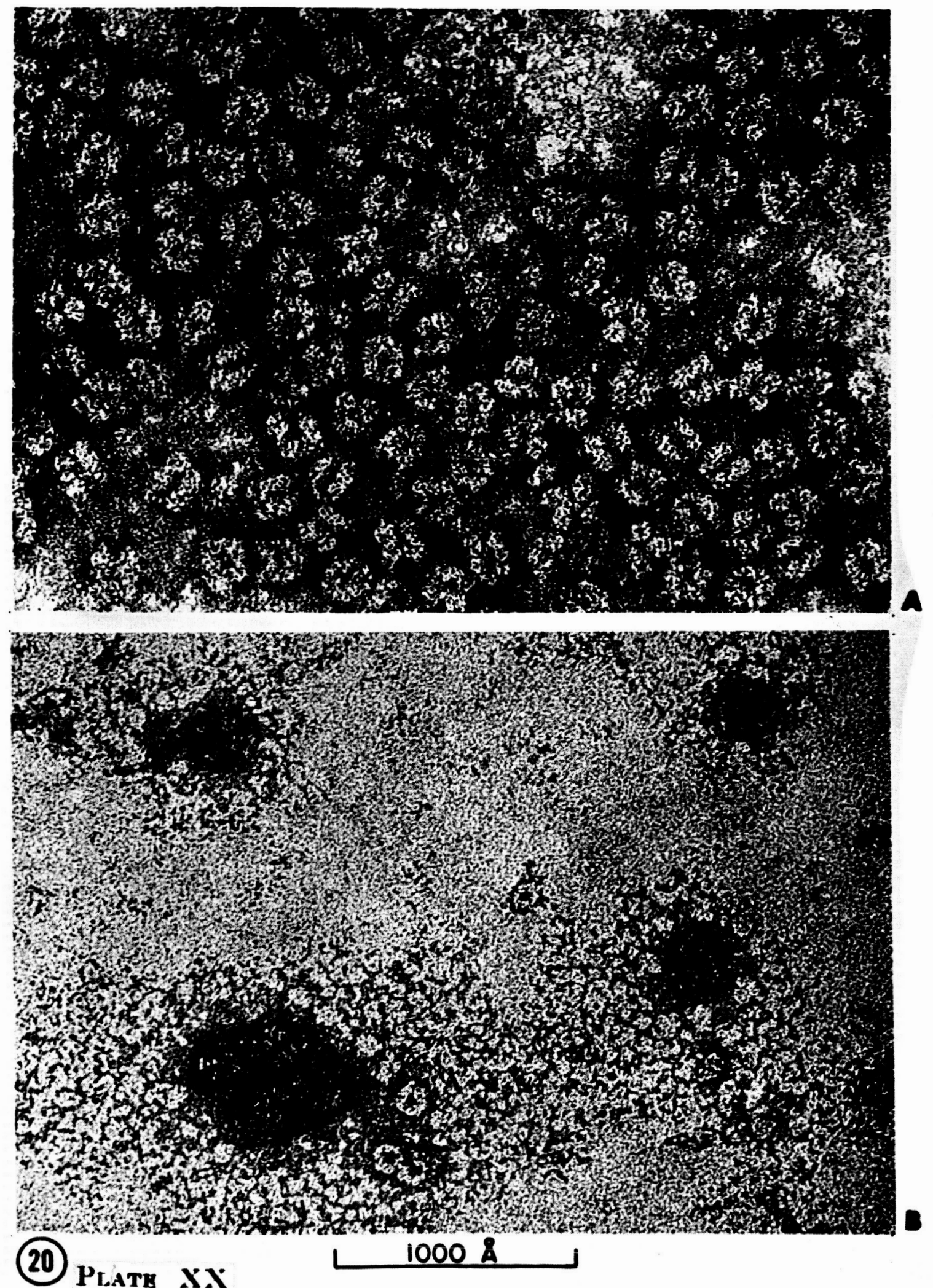

Iimulus polyphemus hemocyanin molecules negatively stained with uranyl acetate. Molecules with the same dimensions, symmetry and substructure as de scribed in Plates XVIII and XIX are observed. (a) X 360,000. (b) X 400,000. 
The smaller squares with a side of $80 \AA$ to $100 \AA$ were earlier identified as the $16 \mathrm{~s}$ component (van Bruggen, 1964). The hexagons (diameter $120 \AA$ to $150 \AA$ ) and the small rectangles of $80 \AA \times 110 \AA$ were shown to be other projections of this $16 \mathrm{~s}$ component. The $24 \mathrm{~s}$ component is seen as a dimer mostly consisting of a hexagon connected with a square or a rectangle. The 6 s component obtained at complete dissociation still shows substructure and measures about $45 \AA$ across.

Staining with uranyl acetate (Plate $X X$ ) shows the same structures again, however, now with much finer detail. This is best seen in the smaller components where the subunits show a very fine substructure that was not visible after staining with phosphotungstate.

Besides the structures described above, a ring structure with a diameter of about $100 \AA$ is regularly seen (Plates XIX, XX). We have considered the possibility that this structure might be an enzyme or another kind of protein present in the Limulus blood; however, this assumption can only be tested by biochemical analysis, and further work remains to be done. The elongated structure of $450 \AA \mathrm{x} 100 \AA$ shown in Plate XIXa probably is a side view of a stack of several of these $100 \AA$ rings. From the periodicity with a spacing of about $65 \AA$ the height of the individual molecules can be estimated to be about $65 \AA$.

No significant differences were found between hemocyanin and apohemocyanin molecules of Limulus (Plate XXI) as examined by a wide variety of positive and negative staining techniques and shadow casting with carbon.

(viii) Homarus americanus

Specimens of hemocyanin of Homarus americanus, negatively stained with PTA (Plate XXII) show pictures which are essentially similar to those described previously by van Bruggen et al. (1963) for other Crustacea. 


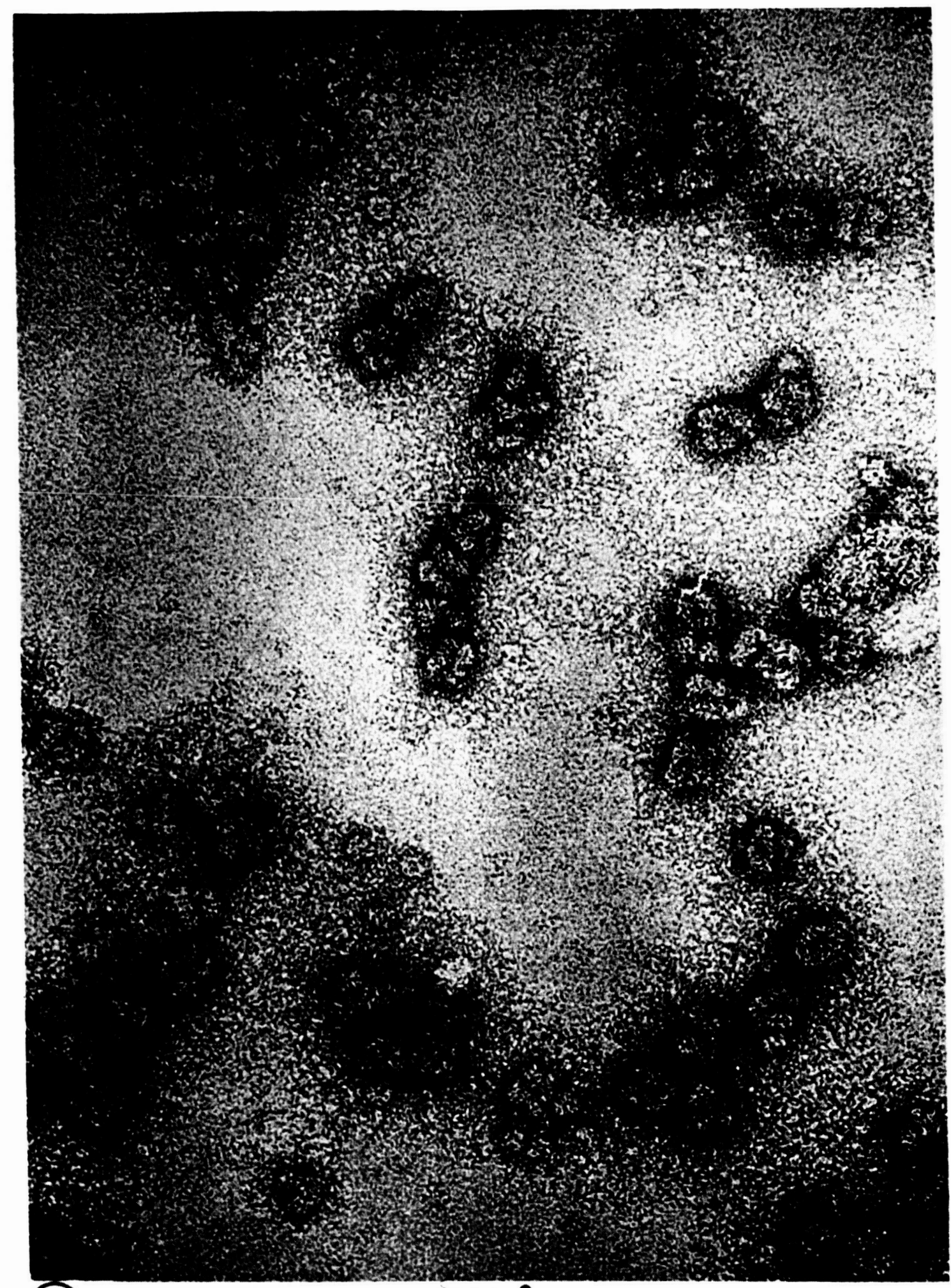

(21) PIATK XXI

$1000 \&$

IImulus polyphemus apohemocyanin molecules sprayed as a mixture with "uranium-EDTA" and then floated on uranyl acetate for about 15 seconds. The same molecular stmuctures are observed as for limulus hemocjanin. X 360,000. 

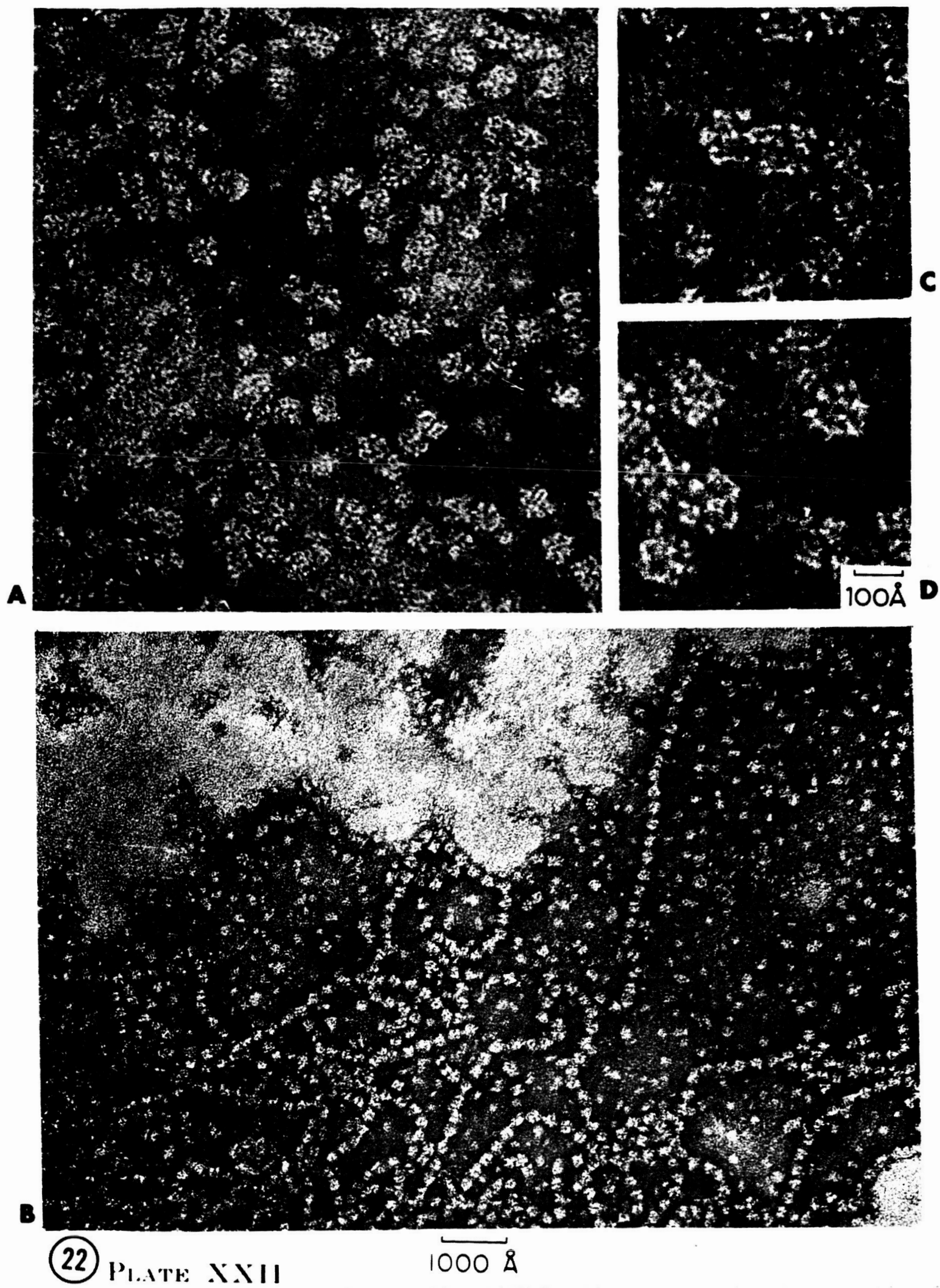

Homarus americanus hemocyanin molecules negatively stained with potassium phosphotungstate. The $16 \mathrm{~s}$ component appears as hexagons with a diameter of $130 \AA$ (d) or as near-rectangles with sides of about $105 \AA$. The $24 \mathrm{~s}$ component is observed as a combination of a hexagon and rectangle (c). An interesting phenomenon found in only one of the hemolymph samples examined was a tendency of the molecules to aggregate laterally into long strings. (b). (a) X 450,000. (b) X 112,500. (d) X 900,000. 
The $16 \mathrm{~s}$ component appears as hexagons with a diameter of $130 \AA$, squares or rectangles with a side of about $105 \AA$. The $24 \mathrm{~s}$ component again appears to be a combination of a hexagon and a square or a rectangle.

The hexagons consist of one, sometimes two, subunits in the center with six around it. Looking at the details, we see that because of the better resolution each of the subunits actually has a ring type structure. This is even better visible after staining with uranyl acetate (Plate XXIII). A substructure for each of these rings is clearly shown.

The same fine structures are observed in the other projection of the $16 \mathrm{~s}$ component.

An interesting phenomena, that only occurred in one of the hemolymph samples, was a tendency of the molecules to aggregate laterally into long strings forming a dense mesh-work (Plate XXIIb).

Preparations of Homarus apohemocyanin (Plate XXIV) were not different in the essential details from those of hemocyanin. 

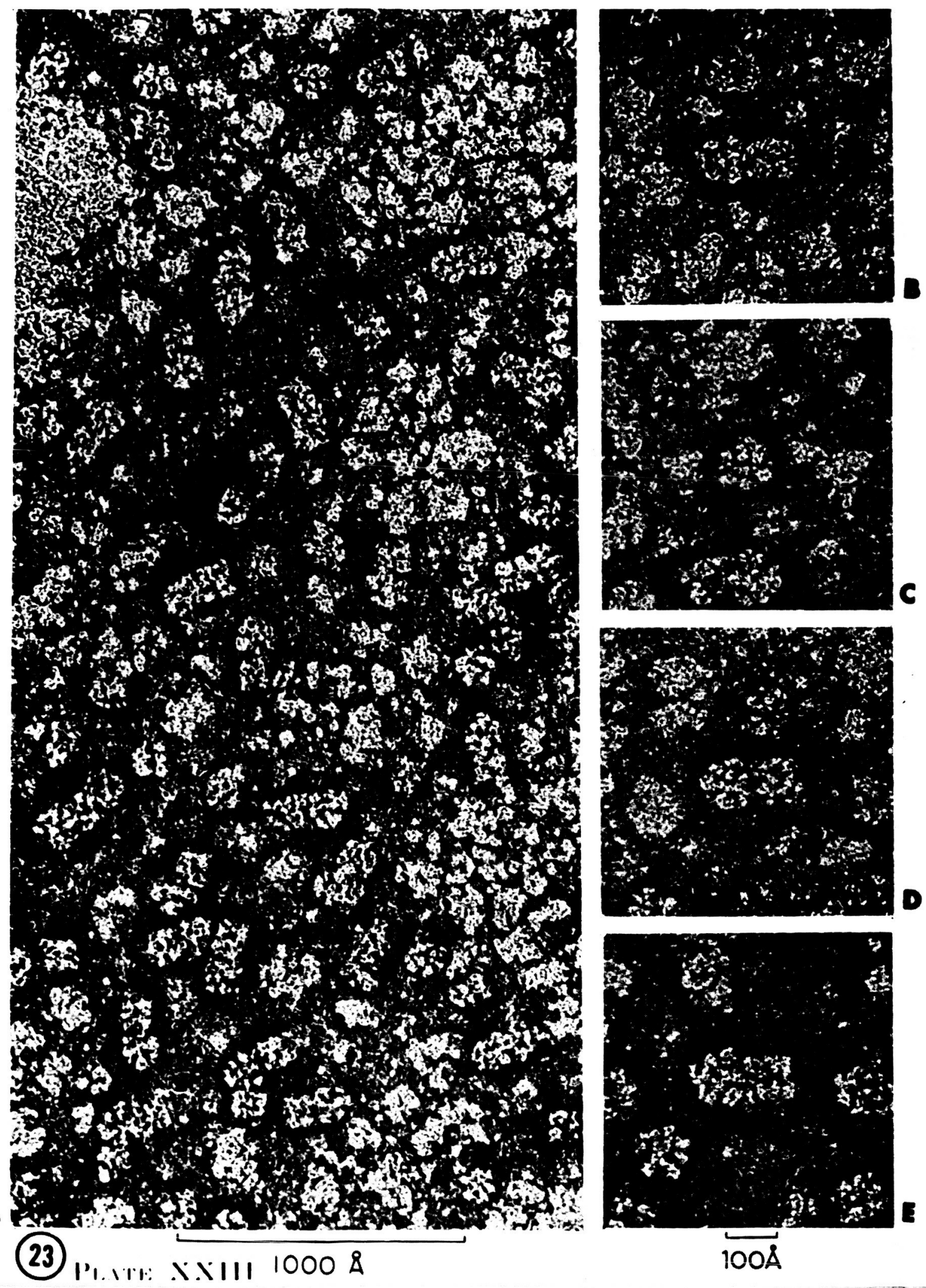

$100 \AA$

PIATE XXIII Homarus americanus hemocyanin molecules negatively stained with uranyl acetate. The same structures are observed as in Plate XXII more clearly. The hexagons consists of one or two subunits in the center with six around it. Each of these subunits has an annular structure; a substructure for each of these rings is clearly shown. (a) X 400,000. (b to e) X 900,000. 

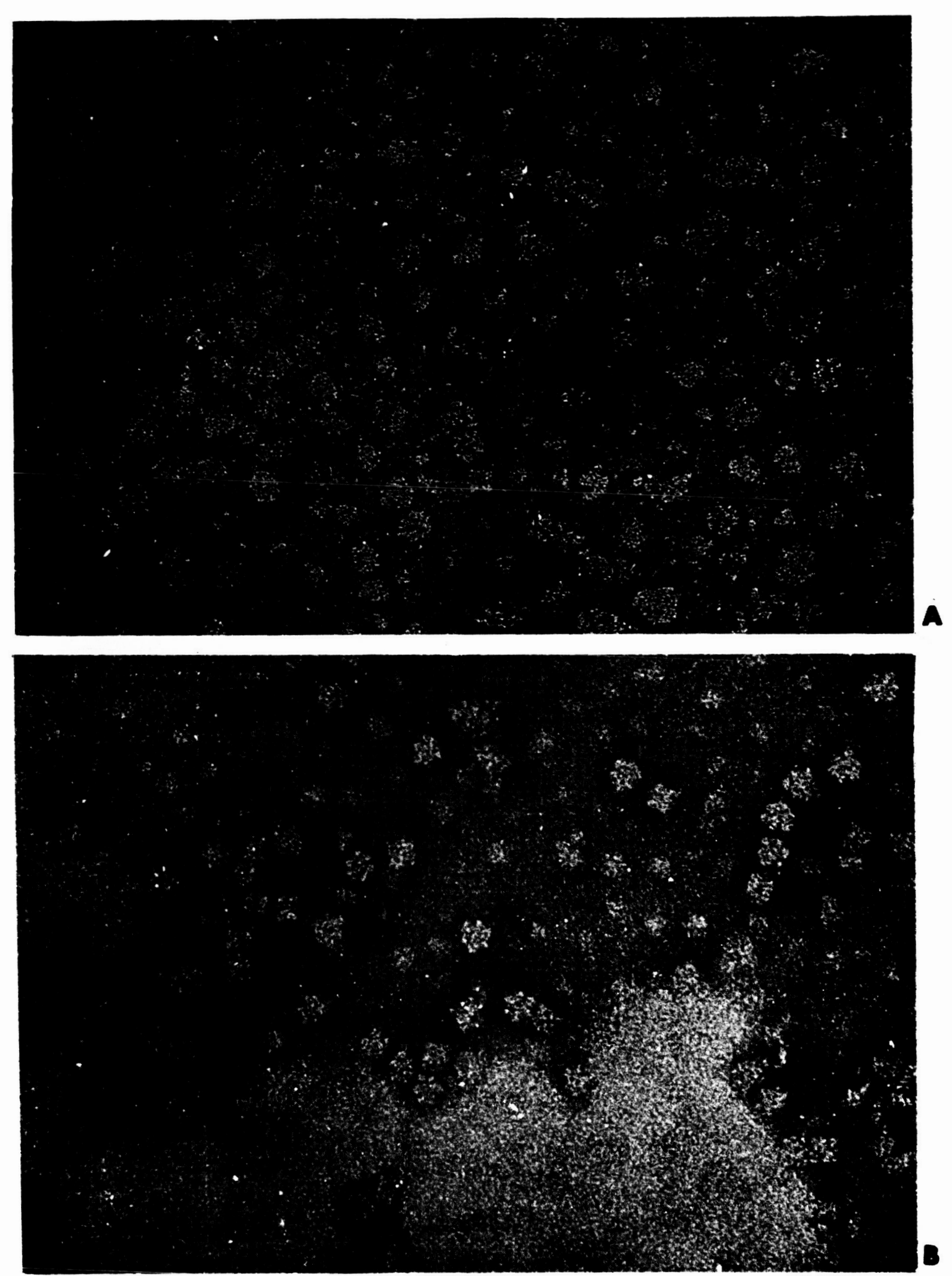

\section{(24) PI..TT: $\times x / V$}

$1000 \AA$

Homarus americanus apohemocyanin molecules negatively stained with uranyl acetate (a) and potassium phosphotungstate (b). Structures with exactly the same dimensions, symmetry, and substructure as described for Homarus hemocyanin are observed. (a)'X 400,000. (b) X 450,000. 


\section{Discussion}

The electron microscopical data presented here for hemocyanin molecules from different biological origins allows us to discuss the analogy between them at a level of resolution close to their quaternary structures. ErikssonQuensel and Svedberg (1936) suggested from sedimentation analysis that a very close structural relation should exist for hemocyanins belonging to the same and to different phylums. They introduced classes for the different components with sedimentation constants ranging from 6 s to $130 \mathrm{~s}$. Their idea that the dissociation would occur into halves, fourths, eights, etc. molecules strongly influenced later workers. Schramm and Berger (1952) proposed from electron micrographs a model for the molecule of Helix pomatia hemocyanin in accordance with this way of dissociation.

Sedimentation analysis showed that the sedimentation constants for the main components of the Arthropoda hemocyanins ranged from $16 \mathrm{~s}$ to $57 \mathrm{~s}$; for the Mollusca hemocyanins from $57 \mathrm{~s}$ to $130 \mathrm{~s}$. The importance of comparing the structure of the $57 \mathrm{~s}$ components from both phylums is obvious. The 57 s components are, in fact, found as main components respectively in Iimulus and Loligo hemocyanin.

Ultracentrifugation analysis showed for the Arthropoda a component with a sedimentation constant of $4 \mathrm{~s}$ to $6 \mathrm{~s}$, as the smallest unit. In agreement with this are the $40 \AA$ to $50 \AA$ structures seen in electron micrographs of dissociated hemocyanins (van Bruggen, 1964). Our present observations show that the structure of these units is roughly annular. At high resolution there are indications of finer substructures (Plates XXII to XXIV). Future biochemical analysis may permit a closer correlation with these observations. The 6 s component is clearly recognized as a building block in the $16 \mathrm{~s}$ component (Plates XXII to XXIV). The structure of 
the $16 \mathrm{~s}$ component is not clearly established. It is outlined as a hexagon, a square, or a rectangle. Levin (1963b) proposed a roughly triagonal prismatic model; van Bruggen et al. (1963) proposed a cube. The $24 \mathrm{~s}$ component is a dimer of the $16 \mathrm{~s}$ component and is mostly seen as a combination of a hexagon with a cube or a rectangle (Plates XXIII to XXIV). In contrast to Levin's (1963c) observations, we found this typical dimer both for Homarus and Limulus belonging respectively to the Crustacea and Merostomata. This emphasizes the relationship between these hemocyanins belonging to the different classes of the same phylum. The 35 s unit, clearly seen as a tetramer of the $16 \mathrm{~s}$ unit, was only observed in Limulus hemocyanin (Plates XVIII to XXI).

The structure of the 57 s unit, also of Limulus hemocyanin, is more difficult to analyze. It shows striking analogy with that of the erythrocruorins (Levin, 1963a). Both are seen as circles and as rectangles consisting of two rows with a gap in between. The exythrocruorins show a very clear hexagonal structure. Limulus hemocyanin sometimes resembles a pentagon. The $57 \mathrm{~s}$ component shows the $24 \mathrm{~s}$ component as an intermediate both during dissociation and reassociation; therefore it might be a pentamer of this $24 \mathrm{~s}$ unit.

Loligo hemocyanin (phylum Mollusca) also has a sedimentation constant of about $57 \mathrm{~s}$. Its structure is completely different from Limulus hemocyanin. This would make unlikely the possibility of a quatemary structural relationship between these hemocyanins belonging to different phylums. Recent ultracentrifugation studies by Van Holde and Cohen (1964a; 1964b) showed a dissociation at high $\mathrm{pH}$ into $11 \mathrm{~s}$ component with a molecular weight of 385,000 . A 19 s component with a molecular weight of 770,000 occurred as an intermediate during this dissociation. The smaller structures visible on Plates XV-XVII probably correspond to those $19 \mathrm{~s}$ and $11 \mathrm{~s}$ components. Sedimentation analysis has not clearly revealed components with a sedimentation constant smaller than $11 \mathrm{~s}$. Electron 
microscopy, however, shows at high pH particles with a diameter of $40 \AA$ to $50 \AA$ that are very similar both in size and structure to the $6 \mathrm{~s}$ component of the Arthropoda hemocyanins. The discrepancy between sedimentation analysis and electron microscopy might be due to a dissociation by the stains of the Il s unit into smaller components. They are shown both in uranyl acetate and phosphotungstate preparations. These $40 \AA$ to $50 \AA$ subunits are clearly the constituent building blocks of Loligo hemocyanin. They are also observed in the circular and rectangular projections of its main component (Plates XVXVII).

When we compare the structures of Ioligo, Helix and Busycon hemocyanin we see circular forms with about the same diameter and symmetry for each of the species and rectangular forms with increasing numbers of rows. This again points out their cylindrical structure. Condie and Langer (1964) suggested for Kelletia kelletia hemocyanin that the circular and rectangular forms represent two different species of molecules. They do not give any proof for this suggestion. We found that the ratio of circles to rectangles can be drastically changed (van Bruggen, 1962) in specimens prepared from a hemocyanin solution before and after treatment with a small amount of non-ionic detergent. This is additional proof that we are dealing here only with a different way of orientation of the same cylindrical molecules. The observation of 20 subunits in the outer ring of the circular forms agrees very well with calculations that give a circular diameter of about $325 \AA$ for 20 subunits, measuring about $45 \AA$ across. For an inner ring of 10 subunits, with a diameter of $45 \AA$, calculations yield a diameter of about $145 \AA$, also in reasonable agreement with our observations.

From a structural point of view Helix hemocyanin can not simply be considered as a dimer of Loligo hemocyanin, and Busycon hemocyanin is more complex than a mere polymer of Helix hemocyanin. Thus, the pattern shown by half Helix molecules (rectangular 3row structure) is different from the corresponding rectangular 
form of the main Loligo component. The Loligo molecule stains evenly, while the half Helix molecule consistently displays asymmetry of staining indicative of a certain polarity. Looking at the 9- and 12-row structures of Busycon hemocyanin (Plates IX - XI) we do not see a simple repetition of the Helix 6-row structure. Rather it appears that one or more 3row structures are attached to the basic 6-row structure characteristic of the Helix hemocyanin. Assuming that these 3-row structures are "closed" at one side and "open" at the other side, we regularly find the "open" side facing the 6row part in the 9- and 12-row stmuctures. This gives rise to the typical staining patterns observed. The same observations were made for structures containing a still larger number of rows. They are always built up from a 6-row main part with the systematic addition of a certain number of 3row structures in the described modular organization patterns.

A critical analysis of the new structural details observed in the central and outer parts of the Helix and Busycon molecules must take into consideration the complex preparation factors Involved. Treatment with uranium compounds and aldehyde fixation plays an important role in the reproducible demonstration of these structures.

The cores of the circular forms of the molecules show patterns ranging from a number of particles disposed in circular arrays, rosettes, (Plates III - XII) to rodlets (Plate XIII). Specimens negatively stained with phosphotungstate show little or no indications of such differentiated structures in the dense cores. The observation of a preferential staining of the central regions of the circular and rectangular forms of hemocyanin and apohemocyanin molecules could be interpreted in essentially two different ways. Either the molecules take up the stain because they are partially hollow inside, or they may contain constituents in the center that strongly interact with the heavy metal stains. Actually, we are dealing here with a combination of negative and positive 
staining. Assuming predominant negative staining we must localize these constituents in the top and bottom layers of the cylindrical molecules, while positive staining would favor localization in the center of the molecules. On the basis of the available data it is difficult to decide clearIy between the two alternative interpretations. However, the possibility of the presence of constituents specifically localized in the core of these molecular complexes is important and warrants further investigation.

The outer coat or layer of about $40 \AA$ to $80 \AA$ which uniformIy surrounds the separate molecules is consistently seen in both unfixed and aldehyde-fixed specimens stained with uranyl acetate (Plates IV - XIII). This outer coat is intimately associated with the dense outer layers of the hemocyanin and apohemocyanin molecules. In through-focal series it exhibits a characteristic uniform fine structure with well-defined borders which is different from the background structure of the carbon film and adjacent uranium particles. In carbon shadowed preparations this outer coat shows a characteristic structure featuring concentric rings with particulate subunits in fairly regular arrangement (Plates V, VI). It is interesting to note that the same type of structure is found in both the circular and rectangular forms of the molecules. The possibility that we may be visualizing a condensed layer of some material which has been displaced from the molecules by preparative manipulations is but one of the many conceivable interpretations. Although the regular occurrence of such a differentiated outer layer is suggestive, we do not know. Whether these structures are actual components of the native molecules, or were produced by our preparation techniques. Nevertheless, the possible presence of a protective coating around these large molecular complexes would have significant biological implications which merit further investigation. 
No significant differences which could be detected by electron microscopy were observed between the hemocyanins and apohemocyanins of the Arthropoda. Certain differences were found when comparing the centers of the circular forms of hemocyanin and apohemocyanin molecules from Helix and Busycon (Plates VIVIII, XI-XIII). Some of these changes may have been caused by the potassium cyanide treatment during copper removal. Further work using micro-incineration, electron diffraction and related techniques combined with biochemical analysis are needed to localize the copper in these molecules. It is very important to know whether hemocyanin is built from equal copper-containing subunits or from copper- and non-copper-containing subunits.

The present studies, which essentially confirm and extend the comprehensive investigations by van Bruggen and collaborators, have mainly served to emphasize the remarkable degree of complexity and structural sophistication of these unique macromolecular complexes. The hemocyanins afford an ideal opportunity for correlated biochemical and physico-chemical studies, since it is now possible to visualize their exquisite fine structure directly at the level of the subunits corresponding closely to the quaternary and perhaps tertiary protein structures. X-ray diffraction studies of hemocyanin crystals may prove to be of key importance in elucidating their molecular organization. Many of the basic problems connected with the organization of multienzyme complexes and other macromolecular assemblies (Schmitt, 1963) will undoubtedly derive valuable methodological and conceptual support from studies in this field.

Finally, it is interesting to recall Redfield's (1933) suggestion that evolution among the hemocyanin bearers has resulted in a great variety of forms, but has led to little advance in the mechanisms for respiration. The different structural variations of the hemocyanins which are now being directly visualized by electron microscopy may well be the 
expression of this evolutionary trend which could not evolve beyond the level of organization of these macromolecular complexes. Although they seem to be built from analogous subunits, hemocyanins from phylum Mollusca are distinctly different from those of phylum Arthropoda. In this connection one could point out the structural analogy between

Limulus hemocyanin and Arenicola (phylum Annelida) hemoglobin, both of which have the same sedimentation constant. Although belonging to different phylums, the evolution of their very different blood proteins resulted in closely related structures. 
We are much indebted to Misses C. M. Runner and D. A. Meddoff for their skillful technical assistance in the specimen preparations, and to Messrs. C. L. Hough, E. Dunker and L. Sherman for expert assistance with all photographic reproductions. Sincere thanks are also due to our colleagues Dr. K. Yada and R. B. Luftig for their many suggestions and kind help in calibration and measurement of the micrographs, and to $\mathrm{Mr}$. L. P. ouwerkerk for important assistance in the instrumentation work. We gratefully acknowledge the kind help of Misses J. E. Richardson and M. A. Arnold in preparing the manuscript. 


\section{REFERENCES}

\section{HEMOCYANIN AND APOHEMOCYANIN ORGANIZATION}

Brenner, S. \& Horne, R. W. (1959). Biochim. biophys. Acta, 34,103 .

Bruggen, E. F. J. van (1962). Thesis. Groningen.

Bruggen, E. F. J. van (1964). Proc. III Eur. Reg. Conf. Electr. Micr., Prague, $\mathrm{B}, 57$.

Bruggen, E. F. J. van, Wiebenga, E. H.\& Gruber, M. (1960). Proc. Eur. Reg. Conf. Electr. Micr., Delft, II, 712.

Bruggen, E. F. J. van, Wiebenga, E. H. \& Gruber, M. (1962a). J. Mol. Biol., 4,1 .

Bruggen, E. F. J. van, Wiebenga, E. H. \& Gruber, M. (1962b). J. Mol. Biol. 4,8 .

Bruggen, E. F. J. van, Schuiten, V., Wiebenga, E. H. \& Gruber, M. (1963). J. Mol. Biol. I, 249.

Caspar, D. L. D. \& Klug, A. (1962). Cold Spr. Harb. Symp. Quant. Biol. 27, 1 .

Clark, G. L., Quaife, M. L., \& Baylor, R. B. (1943). 4, 153. Cohen, L. B. and Van Holde, K. E. (1964). Biochem., 3, 1809. Condie, R. M. \& Langer, R. B. (1964). Science, 144, 1138. Dawson, C. R. \& Mallette, M. F. (1945). Advanc. Protein Chem. 2., 179 .

Eriksson-Quensel, I. \& Svedberg, T. (1936). Biol. Bull. 71, 498. Fernández-Morán, H. (1952). Exp. Cell Res., 3, 282. Fernández-Morán, H. (1953). Exp. Cell Res., 5, 255. Fernández-Morán, H. (1960a). Ann. N. Y. Acad. Sci., 85, 689. Fernández-Morán, H. (1960b). J. Appl. Phys., 31, 1840. Fernández-Morán, H. (1962a). Symp. Internatl. Soc. Cell Biol. $\underline{1}, 411$. 
Fernández-Morán, H. (1962b). Circ., 26, 1039.

Fernández-Morán, H. (1964a). J. Roy. Micr. Soc., 83, 183. Fernández-Morán, H., Blair, P. V. \& Green, D. E. (1964b).

J. Cell Biol. 22, 63.

Fernández-Morán, H. \& Finean, J. P. (1957). J. Biophys.

Biochem. Cyto1., 3, 725.

Fernández-Morán, H., Reed, L. J., Koike, M., \& Willms,

C. R. (1964). Science, 145, 930.

Fernández-Morán, H. \& Schramm, G. (1958). Z. naturf., 136, 68.

Ghiretti, F. (1956). Arch. Biochem. Biophys., 63, 165..

Hall, C. E. (1955). J. Biophys. Biochem. Cytol., I, I.

Huxley, H. E. (1956). Proc. Stockholm Conf. Electr. Micr., 260 .

Huxley, H. E. (1963). J. Mol. Biol., I, 281.

Huxley, H. E. \& Zubay, G. (1960). J. Mol. Biol. 2, 10.

Klotz, I. M. \& Klotz, T. A. (1955). Science, 121, 477.

Kubowitz, F. (1938). Biochem. Z., 299, 32.

Lauffer, M. A. \& Swaby, L. G. (1955). Biol. Bull, 108, 290.

Levin, O. (1963a). J. Mol. Biol., 6, 95.

Levin, 0. (1963b). Arkiv kemi, 21, 1 .

Levin, 0. (1963c). Arkiv kemi, 21, 29.

McLaren, A. D. \& Peterson, G. H. (1961). Nature, 192, 960.

Peterson, R. E. \& Bollier, M. E. (1955). Anal. Chem., 27, 1195.

Polson, A. G. \& Wyckoff, R. W. G. (1947). Nature, 160, 153.

Redfield, A. C. (1934). Biol. Rev. Biol. Proc. Cambridge.

Phil., Soc., 9, 175. 
Roche, J., Bessis, M. \& Thiery, J. P. (1960a). Biochim. biophys. Acta, 41, 182.

Roche, J., Bessis, M. \& Thiery, J. P. (1960b). C. R. Soc. Biol., Paris, 154, 73.

Roche, J., Bessis, M., Breton-Gorius, J. \& Stralin, H. (1961). C. R. Acad. Soi., Paris, 252, 3886.

Sandell, E. B. (1944). Colorimetric Determination of Traces of Metals. 221

Schmitt, F. O. (1963). Develop. Biol., I, 546.

Schramm, G. \& Berger, G. (1952). Z. naturf., 7b, 284.

Stanley, W. \& Anderson, T. (1942). J. Biol. Chem., 146, 25.

Van Holde, K. E. \& Cohen, L. B. (1964a). Biochem., 3, 1803.

Van Holde, K. E. \& Cohen, L. B. (1964b). Brookhaven Symp. Biol. 17, 184 . 